\title{
A HÖLDER-LOGARITHMIC STABILITY ESTIMATE FOR AN INVERSE PROBLEM IN TWO DIMENSIONS
}

\author{
MATTEO SANTACESARIA
}

\begin{abstract}
The problem of the recovery of a real-valued potential in the two-dimensional Schrödinger equation at positive energy from the Dirichlet-to-Neumann map is considered. It is know that this problem is severely ill-posed and the reconstruction of the potential is only logarithmic stable in general. In this paper a new stability estimate is proved, which is explicitly dependent on the regularity of the potentials and on the energy. Its main feature is an efficient increasing stability phenomenon at sufficiently high energies: in some sense, the stability rapidly changes from logarithmic type to Hölder type. The paper develops also several estimates for a non-local Riemann-Hilbert problem which could be of independent interest.
\end{abstract}

\section{INTRODUCTION}

This paper is the last of a series of four papers focusing on stability estimates for the Gel'fand-Calderón problem on the plane. In the first paper, [27], a first global stability estimate is proved. The second and the third paper, [29, 30], deal with stability estimates in the zero-energy and negativeenergy case, respectively, which explicitly depend on the energy and on the regularity of potentials. The present work covers the last and maybe more interesting case when the energy is supposed to be positive.

The Gel'fand-Calderón problem concerns the Schrödinger equation at fixed energy $E$,

$$
(-\Delta+v) \psi=E \psi \quad \text { on } D, \quad E \in \mathbb{R}
$$

where $D$ is a open bounded domain in $\mathbb{R}^{2}$ and $v \in L^{\infty}(D)$ (we will refer to $v$ as a potential). Under the assumption that

(1.2) 0 is not a Dirichlet eigenvalue for the operator $-\Delta+v-E$ in $D$,

1991 Mathematics Subject Classification. 35R30; 35J15.

Key words and phrases. Schrödinger equation, global stability in 2D, increasing stability, positive energy, generalised analytic functions, Riemann-Hilbert problem. 
we can define the Dirichlet-to-Neumann operator $\Phi(E): H^{1 / 2}(\partial D) \rightarrow H^{-1 / 2}(\partial D)$, corresponing to the potential $v$, as follows:

$$
\Phi(E) f=\left.\frac{\partial u}{\partial \nu}\right|_{\partial D}
$$

where $f \in H^{1 / 2}(\partial D), \nu$ is the outer normal of $\partial D$, and $u$ is the $H^{1}(D)$ solution of the Dirichlet problem

$$
(-\Delta+v) u=E u \text { on } D,\left.\quad u\right|_{\partial D}=f .
$$

This construction gives rise to the so-called Gel'fand-Calderón problem.

Problem 1. Given $\Phi(E)$ for a fixed $E \in \mathbb{R}$, find $v$ on $D$.

This problem can be considered as the Gel'fand inverse boundary value problem for the two-dimensional Schrödinger equation at fixed energy (see [11], [20]). At zero energy this problem can also be seen as a generalization of the Calderón problem of the electrical impedance tomography (see [8], [20]).

Note that this problem is not overdetermined, in the sense that we consider the reconstruction of a function $v$ of two variables from inverse problem data dependent on two variables.

In this paper we study interior stability estimates, i.e. we want to prove that given two Dirichlet-to-Neumann operators $\Phi_{1}(E)$ and $\Phi_{2}(E)$, corresponding to potentials $v_{1}$ and $v_{2}$ on $D$, we have that

$$
\left\|v_{1}-v_{2}\right\|_{L^{\infty}(D)} \leq \omega\left(\left\|\Phi_{1}(E)-\Phi_{2}(E)\right\|_{*}\right),
$$

where the function $\omega(t) \rightarrow 0$ as fast as possible as $t \rightarrow 0$ at any fixed $E$ and $\|\cdot\|_{*}$ is some operator norm. The explicit dependence of $\omega$ on $E$ is analysed as well.

There is a wide literature on the Gel'fand-Calderón inverse problem. In the case of complex-valued potentials the global injectivity of the map $v \rightarrow \Phi$ was firstly proved in [20] for $D \subset \mathbb{R}^{d}$ with $d \geq 3$ and in [7] for $d=2$ with $v \in L^{p}$ : in particular, these results were obtained by the use of global reconstructions developed in the same papers. A global logarithmic stability estimate for Problem 1 for $d \geq 3$ was first found by Alessandrini in [1]. In the two-dimensional case the first global stability estimate was given in [27]. In [16] logarithmic stability was proved in dimension $d \geq 2$ without condition (1.2), using more general boundary data (impedance boundary map). For Lipschitz stability estimates concerning this and similar inverse problems with finite dimensional restrictions see [2], [3], [5] and [6]. 
In [29] and in [30] we considered Problem 1 at zero and negative energy, respectively, and answered the following question: how the stability estimates vary with respect to the smoothness of the potentials and the energy.

This paper completes the preceding works by considering the positive energy case.

We will assume for simplicity that

$$
\begin{array}{ll}
D \text { is an open bounded domain in } \mathbb{R}^{2}, & \partial D \in C^{2}, \\
v \in W^{m, 1}\left(\mathbb{R}^{2}\right) \text { for some } m>2, \quad \bar{v}=v, & \operatorname{supp} v \subset D,
\end{array}
$$

where

$$
\begin{aligned}
& W^{m, 1}\left(\mathbb{R}^{2}\right)=\left\{v: \partial^{J} v \in L^{1}\left(\mathbb{R}^{2}\right),|J| \leq m\right\}, \quad m \in \mathbb{N} \cup\{0\}, \\
& J \in(\mathbb{N} \cup\{0\})^{2}, \quad|J|=J_{1}+J_{2}, \quad \partial^{J} v(x)=\frac{\partial^{|J|} v(x)}{\partial x_{1}^{J_{1}} \partial x_{2}^{J_{2}}} .
\end{aligned}
$$

Let

$$
\|v\|_{m, 1}=\max _{|J| \leq m}\left\|\partial^{J} v\right\|_{L^{1}\left(\mathbb{R}^{2}\right)} .
$$

We will need the following regularity condition:

$$
E>E_{1}
$$

where $E_{1}=E_{1}\left(\|v\|_{m, 1}, D\right)$ or, roughly speaking, $E$ is sufficiently great with respect to some appropriate norm of the potential. This condition implies, in particular, that the Faddeev eigenfunctions are well-defined on the entire fixed-energy surface in the spectral parameter (see Section 2 and Remark 2.1).

Theorem 1.1. Let the conditions (1.2), (1.5), (1.7) hold for the potentials $v_{1}, v_{2}$, where $D$ is fixed, and let $\Phi_{1}(E), \Phi_{2}(E)$ be the corresponding Dirichletto-Neumann operators at fixed positive energy $E>0$. Let $\left\|v_{j}\right\|_{m, 1} \leq N$, $j=1,2$, for some $N>0$. Then there exists a constant $C_{1}=C_{1}(D, N, m)$ such that for any $0<\tau \leq 1$, we have

$$
\left\|v_{2}-v_{1}\right\|_{L^{\infty}(D)} \leq C_{1}\left(E \delta^{\tau}+\left(\sqrt{E}+(1-\tau) \log \left(3+\delta^{-1}\right)\right)^{-(m-2)}\right)
$$

for every $\delta<\tilde{\delta}(\tau)$, where $\delta=\left\|\Phi_{2}(E)-\Phi_{1}(E)\right\|_{L^{\infty}(\partial D) \rightarrow L^{\infty}(\partial D)}$.

This results yields the following corollary.

Corollary 1.2. Under the same assumptions, there exists a constant $C_{2}=$ $C_{2}(E, D, N, m)$ such that

$$
\left\|v_{2}-v_{1}\right\|_{L^{\infty}(D)} \leq C_{2}\left(\log \left(3+\delta^{-1}\right)\right)^{-\alpha}, \quad \alpha=m-2,
$$

for $\delta<\tilde{\delta}$. 
The novelty of estimate (1.9), with respect to [27], is that, as $m \rightarrow+\infty$, we have $\alpha \rightarrow+\infty$. Moreover, under the assumption of Theorem 1.1. according to instability estimates of Mandache [19] and Isaev [13, our result is almost sharp. To be more precise, it was proved that stability estimate (1.9) cannot hold for $\alpha>2 m$ for $C^{m}$ real-valued potentials and $\alpha>m$ for $C^{m}$ complex-valued potentials. Note that stability estimates and instability counterexamples are proved in different function spaces. In particular, by Sobolev embedding, we have only that $W^{m, 1+\varepsilon}(D) \subset C^{m-2}(D)$ for any $\varepsilon>0$. From this and the fact that the same stability holds in the linearized case (Born approximation, see [26]), we believe that our result is in fact sharp. Unfortunately we could not find yet an explicit counterexample in the $W^{m, 1}$ class. Our estimates are still valid for complex-valued potentials, if $E$ is sufficiently large with respect to $\|v\|_{C(\bar{D})}$ : in this case we can't use the formulas at the beginning of Section 4 for the solution of the RiemannHilbert problem and thus it is necessary to follow a more general approach, like in [21, §6].

Estimate (1.9) also extends the result obtained in [29] for the same problem at zero energy and in [30] at negative energy. In dimension $d \geq 3$ a global stability estimate similar to (1.9) was proved in 25], at zero energy.

As regards (1.8), its main feature is the explicit dependence on the energy $E$. This estimate consist of two parts, the first logarithmic and the second Hölder; when $E$ increases, the logarithmic part decreases and the Hölder part becomes dominant. This estimate is sharp not only with respect to the dependence on the smoothness of the potentials, but also with respect to the energy, as shown in [14]. It extends the result of [30, where a similar energydependent stability estimate was obtained at negative energy. Yet in that case the Hölder part grows exponentially with the energy, while in the present work it grows linearly. For this reason estimate (1.8), namely when $\tau=$ 1 , is totally coherent with the Lipschitz stable approximate reconstruction algorithms developed in [22] and [28].

Estimate (1.8) is the first stability result in two dimensions for the Gel'fandCalderón problem at positive energy with an explicit dependence on the smoothness of potential and on the energy. In dimension $d \geq 3$, global energy-dependent stability estimates changing from logarithmic type to Lipschitz type at high energies were given in [18] and greatly improved in [17]. In turn, the paper [18] was preceeded by [24]. See also [15] for similar estimates for another inverse boundary value problem.

The proof of Theorem 1.1 follows the scheme of [30] and it is based on $\bar{\partial}$ techniques. The map $\Phi(E) \rightarrow v(x)$ is considered as the composition of $\Phi(E) \rightarrow\left(r(\lambda), \rho\left(\lambda, \lambda^{\prime}\right)\right)$ and $\left(r(\lambda), \rho\left(\lambda, \lambda^{\prime}\right)\right) \rightarrow v(x)$, where $r(\lambda)$ and $\rho\left(\lambda, \lambda^{\prime}\right)$ 
are complex valued functions, closely related to the so-called generalised scattering amplitude (see Section 2 for details).

The stability of $\Phi(E) \rightarrow\left(r(\lambda), \rho\left(\lambda, \lambda^{\prime}\right)\right)$ - previously known only for $E \leq 0$ - relies on some identities of [23] (based in particular on [1]), and estimates on $r(\lambda)$ for $\lambda$ near 0 and $\infty$. The reconstrution of $r(\lambda)$ from $\Phi(E)$ is logarithmic stable with respect to $\Phi$ (at fixed $E$ ), while the reconstruction of $\rho\left(\lambda, \lambda^{\prime}\right)$ is Lipschitz stable. These results are proved in section 3 ,

The stability of $\left(r(\lambda), \rho\left(\lambda, \lambda^{\prime}\right)\right) \rightarrow v(x)$ is of Hölder type and it is proved in section 4. This is the most challenging part of the paper because we need to establish several new estimates for the non-local Riemann-Hilbert problem solved by $r(\lambda)$ and $\rho\left(\lambda, \lambda^{\prime}\right)$ (see Section 2 for details). We make great use of the theory of generalised analytic functions of Ahlfors-Vekua and the main reference is [31. In particular, we establish pointwise and $L^{p}$ estimates for solutions of non-homogeneous $\bar{\partial}$-equations with pole singularities.

In Section 5 we show how the composition of the two above-mentioned maps gives the result of Theorem 1.1 .

\section{Preliminaries}

We recall the definition of the Faddeev eigenfunctions $\psi(x, k)$ of equation (1.1), for $x=\left(x_{1}, x_{2}\right) \in \mathbb{R}^{2}, k=\left(k_{1}, k_{2}\right) \in \Sigma_{E} \subset \mathbb{C}^{2}, \Sigma_{E}=\left\{k \in \mathbb{C}^{2}: k^{2}=\right.$ $\left.k_{1}^{2}+k_{2}^{2}=E\right\}$ for $E \neq 0$ (see [9], [21]). We first extend $v \equiv 0$ on $\mathbb{R}^{2} \backslash D$ and define $\psi(x, k)$ as the solution of the following integral equation:

$$
\begin{aligned}
& \psi(x, k)=e^{i k x}+\int_{y \in \mathbb{R}^{2}} G(x-y, k) v(y) \psi(y, k) d y, \\
& G(x, k)=g(x, k) e^{i k x}, \\
& g(x, k)=-\left(\frac{1}{2 \pi}\right)^{2} \int_{\xi \in \mathbb{R}^{2}} \frac{e^{i \xi x}}{\xi^{2}+2 k \xi} d \xi,
\end{aligned}
$$

where $x \in \mathbb{R}^{2}, k \in \Sigma_{E} \backslash \mathbb{R}^{2}$. It is convenient to write (2.1) in the following form

$$
\mu(x, k)=1+\int_{y \in \mathbb{R}^{2}} g(x-y, k) v(y) \mu(y, k) d y,
$$

where $\mu(x, k) e^{i k x}=\psi(x, k)$.

For $\operatorname{Im} k=0$ formulas (2.1)-(2.4) make no sense; however, the following limits make sense

$$
\begin{gathered}
\psi_{\gamma}(x, k)=\psi(x, k+i 0 \gamma), \quad G_{\gamma}(x, k)=G(x, k+i 0 \gamma), \\
\mu_{\gamma}(x, k)=\mu(x, k+i 0 \gamma),
\end{gathered}
$$


We define $\mathcal{E}_{E} \subset \Sigma_{E} \backslash \mathbb{R}^{2}$ the set of exceptional points of integral equation (2.4): $k \in \Sigma_{E} \backslash\left(\mathcal{E}_{E} \cup \mathbb{R}^{2}\right)$ if and only if equation (2.4) is uniquely solvable in $L^{\infty}\left(\mathbb{R}^{2}\right)$.

Remark 2.1. From [22, Proposition 1.1] we have that there exists $E_{0}=$ $E_{0}\left(\|v\|_{m, 1}, D\right)$ such that for $|E| \geq E_{0}\left(\|v\|_{m, 1}, D\right)$ there are no exceptional points for equation (2.4), i.e. $\mathcal{E}_{E}=\emptyset$ : thus the Faddeev eigenfunctions exist (unique) for all $k \in \Sigma_{E} \backslash \mathbb{R}^{2}$.

Following [12], [21], we make the change of variables

$$
\begin{array}{cl}
z=x_{1}+i x_{2}, & \lambda=\frac{k_{1}+i k_{2}}{\sqrt{E}}, \\
k_{1}=\left(\lambda+\frac{1}{\lambda}\right) \frac{\sqrt{E}}{2}, & k_{2}=\left(\frac{1}{\lambda}-\lambda\right) \frac{i \sqrt{E}}{2},
\end{array}
$$

and write $\psi, \mu$ as functions of these new variables. For $|\lambda|=1$ and $E>0$ formulas (2.3) and (2.4) make no sense but the following limits do:

$$
\begin{gathered}
\psi_{ \pm}(z, \lambda)=\psi(z, \lambda(1 \mp 0)), \quad \mu_{ \pm}(z, \lambda)=\psi(z, \lambda(1 \mp 0)), \\
g_{ \pm}(z, \lambda)=g(z, \lambda(1 \mp 0)) .
\end{gathered}
$$

For $k \in \Sigma_{E} \backslash\left(\mathcal{E}_{E} \cup \mathbb{R}^{2}\right)$ we can define, for the corresponding $\lambda$, the following generalised scattering amplitude,

$$
\begin{aligned}
b(\lambda, E)= & \frac{1}{(2 \pi)^{2}} \int_{\mathbb{C}} \exp \left[\frac{i}{2} \sqrt{E}\left(1+(\operatorname{sgn} E) \frac{1}{\lambda \bar{\lambda}}\right)\right. \\
& \times((\operatorname{sgn} E) z \bar{\lambda}+\lambda \bar{z})] v(z) \mu(z, \lambda) d \operatorname{Re} z d \operatorname{Im} z,
\end{aligned}
$$

and the functions $h_{ \pm}$,

$$
\begin{aligned}
h_{ \pm}\left(\lambda, \lambda^{\prime}, E\right)= & \left(\frac{1}{2 \pi}\right)^{2} \int_{\mathbb{C}} \exp \left[-\frac{i}{2} \sqrt{E}\left(\lambda^{\prime} \bar{z}+z / \lambda^{\prime}\right)\right] \\
& \times v(z) \psi_{ \pm}(z, \lambda) d \operatorname{Re} z d \operatorname{Im} z
\end{aligned}
$$

for $|\lambda|=\left|\lambda^{\prime}\right|=1$. It is useful to introduce the following auxiliary functions $h_{1}, h_{2}$,

$$
\begin{aligned}
h_{1}\left(\lambda, \lambda^{\prime}\right)= & \theta\left[-\frac{1}{i}\left(\frac{\lambda^{\prime}}{\lambda}-\frac{\lambda}{\lambda^{\prime}}\right)\right] h_{+}\left(\lambda, \lambda^{\prime}\right) \\
& -\theta\left[\frac{1}{i}\left(\frac{\lambda^{\prime}}{\lambda}-\frac{\lambda}{\lambda^{\prime}}\right)\right] h_{-}\left(\lambda, \lambda^{\prime}\right), \\
h_{2}\left(\lambda, \lambda^{\prime}\right)= & \theta\left[-\frac{1}{i}\left(\frac{\lambda^{\prime}}{\lambda}-\frac{\lambda}{\lambda^{\prime}}\right)\right] h_{-}\left(\lambda, \lambda^{\prime}\right) \\
& -\theta\left[\frac{1}{i}\left(\frac{\lambda^{\prime}}{\lambda}-\frac{\lambda}{\lambda^{\prime}}\right)\right] h_{+}\left(\lambda, \lambda^{\prime}\right),
\end{aligned}
$$



and $\rho$, solution of the following integral equations,

$$
\begin{aligned}
& \rho\left(\lambda, \lambda^{\prime}\right)+\pi i \int_{\left|\lambda^{\prime \prime}\right|=1} \rho\left(\lambda, \lambda^{\prime \prime}\right) \theta\left[\frac{1}{i}\left(\frac{\lambda^{\prime}}{\lambda^{\prime \prime}}-\frac{\lambda^{\prime \prime}}{\lambda^{\prime}}\right)\right] \\
& \times h_{1}\left(\lambda^{\prime \prime}, \lambda^{\prime}\right)\left|d \lambda^{\prime \prime}\right|=-\pi i h_{1}\left(\lambda, \lambda^{\prime}\right), \\
& \rho\left(\lambda, \lambda^{\prime}\right)+\pi i \int_{\left|\lambda^{\prime \prime}\right|=1} \rho\left(\lambda, \lambda^{\prime \prime}\right) \theta\left[-\frac{1}{i}\left(\frac{\lambda^{\prime}}{\lambda^{\prime \prime}}-\frac{\lambda^{\prime \prime}}{\lambda^{\prime}}\right)\right] \\
& \times h_{2}\left(\lambda^{\prime \prime}, \lambda^{\prime}\right)\left|d \lambda^{\prime \prime}\right|=-\pi i h_{2}\left(\lambda, \lambda^{\prime}\right),
\end{aligned}
$$

for $|\lambda|=\left|\lambda^{\prime}\right|=1$. Here and in the following we drop the dependence of some functions on $E$ for simplicity's sake.

The functions just defined play an important role in the following RiemannHilbert problem solved by $\mu$. When $v$ is real-valued and $E>0$ we have (see [21] for more details):

$$
\frac{\partial}{\partial \bar{\lambda}} \mu(z, \lambda)=r(z, \lambda) \overline{\mu(z, \lambda)}
$$

for $\lambda$ not an exceptional point (i.e. $k(\lambda) \in \Sigma_{E} \backslash\left(\mathcal{E}_{E} \cup \mathbb{R}^{2}\right)$ ) and $|\lambda| \neq 1$, where

$$
\begin{aligned}
r(z, \lambda) & =r(\lambda) \exp \left[-\frac{i}{2} \sqrt{E}\left(1+(\operatorname{sgn} E) \frac{1}{\lambda \bar{\lambda}}\right)(z \bar{\lambda}+\lambda \bar{z})\right], \\
r(\lambda) & =\frac{\pi}{\bar{\lambda}} \operatorname{sgn}(\lambda \bar{\lambda}-1) b(\lambda, E),
\end{aligned}
$$

where $b$ is defined in (2.9);

$$
\mu_{+}(z, \lambda)=\mu_{-}(z, \lambda)+\int_{\left|\lambda^{\prime}\right|=1} \rho\left(\lambda, \lambda^{\prime}, z\right) \mu_{-}\left(z, \lambda^{\prime}\right)\left|d \lambda^{\prime}\right|
$$

for $|\lambda|=1$, where

$$
\rho\left(\lambda, \lambda^{\prime}, z\right)=\rho\left(\lambda, \lambda^{\prime}\right) \exp \left[\frac{i \sqrt{E}}{2}\left(\left(\lambda^{\prime}-\lambda\right) \bar{z}+\left(\frac{1}{\lambda^{\prime}}-\frac{1}{\lambda}\right) z\right)\right],
$$

where $\rho\left(\lambda, \lambda^{\prime}\right)$ is defined in (2.13). In addition we have

$$
\begin{gathered}
\lim _{|\lambda| \rightarrow \infty} \mu(z, \lambda)=1, \\
\mu(z, \lambda)=1+\mu_{-1}(z) \lambda^{-1}+o\left(|\lambda|^{-1}\right), \quad \text { for }|\lambda| \rightarrow \infty \\
v(z)=2 i \sqrt{E} \frac{\partial}{\partial z} \mu_{-1}(z) .
\end{gathered}
$$


We recall that if $v \in W^{m, 1}\left(\mathbb{R}^{2}\right)$ with $\operatorname{supp} v \subset D$, then $\|\hat{v}\|_{\alpha, m}<+\infty$ for some $0<\alpha<1$, where

$$
\begin{gathered}
\hat{v}(p)=(2 \pi)^{-2} \int_{\mathbb{R}^{2}} e^{i p x} v(x) d x, \quad p \in \mathbb{C}^{2}, \\
\|u\|_{\alpha, m}=\left\|\left(1+|p|^{2}\right)^{m / 2} u(p)\right\|_{\alpha}, \\
\|w\|_{\alpha}=\sup _{p, \xi \in \mathbb{R}^{2},|\xi| \leq 1}\left(|w(p)|+|\xi|^{-\alpha}|w(p+\xi)-w(p)|\right),
\end{gathered}
$$

for test functions $u, w$.

We restate in an adapted form a lemma from [22, Lemma 2.1].

Lemma 2.1. Let the conditions (1.5), (1.7) hold for a potentials $v$. Let $\mu(x, k)$ be the associated Faddeev functions. Then, for any $0<\sigma<1$, we have

$$
|\mu(x, k)-1|+\left|\frac{\partial \mu(x, k)}{\partial x_{1}}\right|+\left|\frac{\partial \mu(x, k)}{\partial x_{2}}\right| \leq|\operatorname{Re} k|^{-\sigma} c(m, \sigma)\|\hat{v}\|_{\alpha, m},
$$

for $k \in \mathbb{C}^{2} \backslash \mathbb{R}^{2}$

$$
\left|\mu_{\gamma}(x, k)-1\right|+\left|\frac{\partial \mu_{\gamma}(x, k)}{\partial x_{1}}\right|+\left|\frac{\partial \mu_{\gamma}(x, k)}{\partial x_{2}}\right| \leq|k|^{-\sigma} c(m, \sigma)\|\hat{v}\|_{\alpha, m},
$$

for $k \in \mathbb{R}^{2}, \gamma \in S^{1}$. In both cases we suppose also that $k^{2} \geq R$, where $R$ is defined in Lemma 2.2.

The following lemma is a variation of a result in 22 and it is proved in [30].

Lemma 2.2. Let the conditions (1.5), (1.7) hold for a potentials $v$ and let $E \in \mathbb{R} \backslash\{0\}$. Then there exists an $R=R\left(m,\|\hat{v}\|_{\alpha, m}\right)>1$, such that

$$
|b(\lambda, E)| \leq 2\|\hat{v}\|_{\alpha, m}\left(1+|E|(|\lambda|+\operatorname{sgn}(E) /|\lambda|)^{2}\right)^{-m / 2},
$$

for $|\lambda|>\frac{2 R}{|E|^{1 / 2}}$ and $|\lambda|<\frac{|E|^{1 / 2}}{2 R}$

Let us mention that Lemma 2.2 of [29] and Lemma 2.1 of [30] should be corrected using the norm $\|\cdot\|_{\alpha, m}$ instead of $\|\cdot\|_{m}$.

We also restate [4, Lemma 2.6].

Lemma 2.3 ([4]). Let $q_{1} \in L^{s_{1}}(\mathbb{C}) \cap L^{s_{2}}(\mathbb{C}), 1<s_{1}<2<s_{2}<\infty$ and $q_{2} \in$ $L^{s}(\mathbb{C}), 1<s<2$. Assume $u$ is a function in $L^{\tilde{s}}(\mathbb{C})$, with $1 / \tilde{s}=1 / s-1 / 2$, which satisfies

$$
\frac{\partial u(\lambda)}{\partial \bar{\lambda}}=q_{1}(\lambda) \bar{u}(\lambda)+q_{2}(\lambda), \quad \lambda \in \mathbb{C} .
$$

Then there exists $c=c\left(s, s_{1}, s_{2}\right)>0$ such that

$$
\|u\|_{L^{\tilde{s}}} \leq c\left\|q_{2}\right\|_{L^{s}} \exp \left(c\left(\left\|q_{1}\right\|_{L^{s_{1}}}+\left\|q_{1}\right\|_{L^{s_{2}}}\right)\right) .
$$


We will make also use of the well-known Hölder's inequality, which we recall in a special case: for $f \in L^{p}(\mathbb{C}), g \in L^{q}(\mathbb{C})$ such that $1 \leq p, q \leq \infty$, $1 \leq r<\infty, 1 / p+1 / q=1 / r$, we have

$$
\|f g\|_{L^{r}(\mathbb{C})} \leq\|f\|_{L^{p}(\mathbb{C})}\|g\|_{L^{q}(\mathbb{C})} .
$$

Throughout all the paper $c(\alpha, \beta, \ldots)$ is a positive constant depending on parameters $\alpha, \beta, \ldots$

\section{From $\Phi(E)$ TO $r(\lambda)$ AND $\rho\left(\lambda, \lambda^{\prime}\right)$}

We begin recalling a lemma from [30], which we restate in the case $E>0$.

Lemma 3.1. Let the conditions (1.5), (1.7) hold and take $0<a_{1} \leq \min \left(1, \frac{|E|^{1 / 2}}{2 R}\right)$, $a_{2} \geq \max \left(1, \frac{2 R}{|E|^{1 / 2}}\right)$, for $E>0$ and $R$ as defined in Lemma 2.2. Then for $p \geq 1$ we have

$$
\begin{aligned}
& \left\||\lambda|^{j} r(\lambda)\right\|_{L^{p}\left(|\lambda|<a_{1}\right)} \leq c(p, m)\|\hat{v}\|_{\alpha, m}|E|^{-m / 2} a_{1}^{m-1+j+2 / p}, \\
& \left\||\lambda|^{j} r(\lambda)\right\|_{L^{p}\left(|\lambda|>a_{2}\right)} \leq c(p, m)\|\hat{v}\|_{\alpha, m}|E|^{-m / 2} a_{2}^{-m-1+j+2 / p},
\end{aligned}
$$

where $j=1,0,-1$ and $r$ was defined in (2.16).

Note that, in contrast to the case $E<0$, this Lemma holds even when $a_{1}=a_{2}=1$, thanks to the sign in Lemma 2.2.

The following Lemma extends [30, Lemma 3.2] to the positive energy case.

Lemma 3.2. Let $D \subset\left\{x \in \mathbb{R}^{2}:|x| \leq l\right\}, E>0, v_{1}, v_{2}$ be two potentials satisfying (1.2), (1.5), (1.7), $\Phi_{1}(E), \Phi_{2}(E)$ the corresponding Dirichlet-toNeumann operator and $b_{1}, b_{2}$ the corresponding generalised scattering amplitude. Let $\left\|v_{j}\right\|_{m, 1} \leq N, j=1,2$. Then we have

$$
\left|b_{2}(\lambda)-b_{1}(\lambda)\right| \leq c(D, N, m) \exp \left[l \sqrt{|E|}|| \lambda\left|-\frac{1}{|\lambda|}\right|\right]\left\|\Phi_{2}(E)-\Phi_{1}(E)\right\|_{*},
$$

for $\lambda \neq 0$, where $\|\cdot\|_{*}=\|\cdot\|_{L^{\infty}(\partial D) \rightarrow L^{\infty}(\partial D)}$.

Proof. We have the following identity:

$$
b_{2}(\lambda)-b_{1}(\lambda)=\left(\frac{1}{2 \pi}\right)^{2} \int_{\partial D} \psi_{1}(x, \overline{k(\lambda)})\left(\Phi_{2}(E)-\Phi_{1}(E)\right) \psi_{2}(x, k(\lambda)) d x
$$

where $\psi_{i}(x, k)$ are the Faddeev functions associated to the potential $v_{i}, i=$ 1,2. This identity is a particular case of the one in [23, Theorem 1]: we refer to that paper for a proof. 
From this identity we obtain:

$$
\left|b_{2}(\lambda)-b_{1}(\lambda)\right| \leq \frac{1}{(2 \pi)^{2}}\left\|\psi_{1}(\cdot, k)\right\|_{L^{\infty}(\partial D)}\left\|\Phi_{2}(E)-\Phi_{1}(E)\right\|_{*}\left\|\psi_{2}(\cdot, k)\right\|_{L^{\infty}(\partial D)} .
$$

Now using Lemma 2.1 and the change of variables in Section 2, we get

$$
\begin{aligned}
& \left\|\psi_{j}(\cdot, k(\lambda))\right\|_{L^{\infty}(\partial D)} \leq\left\|e^{\frac{i \sqrt{E}}{2}(\bar{z} \lambda+z / \lambda)} \mu_{j}(\cdot, k(\lambda))\right\|_{L^{\infty}(\partial D)} \\
& \quad \leq e^{\frac{\sqrt{E}}{2} l|\lambda-1 / \bar{\lambda}|}\left\|\mu_{j}(\cdot, k(\lambda))\right\|_{L^{\infty}(\partial D)} \\
& \quad \leq e^{\frac{\sqrt{E}}{2} l|\lambda|-\mid 1 / \lambda \|}\left(\left\|\mu_{j}(\cdot, k(\lambda))-1\right\|_{L^{\infty}(\partial D)}+\|1\|_{L^{\infty}(\partial D)}\right) \\
& \quad \leq c(D, N, m) e^{\frac{\sqrt{E}}{2} l|| \lambda|-| 1 / \lambda \mid \|},
\end{aligned}
$$

for $j=1,2$. This, combined with (3.5), gives (3.3).

The following proposition shows that the map $\Phi(E) \rightarrow \rho\left(\lambda, \lambda^{\prime}\right)$ is Lipschitz stable.

Proposition 3.3. Let $D \subset\left\{x \in \mathbb{R}^{2}:|x| \leq l\right\}, E>0, v_{1}, v_{2}$ be two potentials satisfying (1.2), (1.5), (1.7), $\Phi_{1}(E), \Phi_{2}(E)$ the corresponding Dirichletto-Neumann operator and $\rho_{1}, \rho_{2}$ the corresponding functions as defined in (2.13). Let $\left\|v_{j}\right\|_{m, 1} \leq N, j=1,2$. Then we have

$$
\left\|\rho_{2}-\rho_{1}\right\|_{L^{2}(T \times T)} \leq c(D, N, m)\left\|\Phi_{2}(E)-\Phi_{1}(E)\right\|_{*},
$$

for $E \geq E_{2}=E_{2}(N, m)$, where $T=\{\lambda \in \mathbb{C}:|\lambda|=1\}$ and $\|\cdot\|_{*}=$ $\|\cdot\|_{L^{\infty}(\partial D) \rightarrow L^{\infty}(\partial D)}$.

Proof. We begin proving

$$
\left\|f_{2}-f_{1}\right\|_{L^{2}(T \times T)} \leq c(D, N, m)\left\|\Phi_{2}(E)-\Phi_{1}(E)\right\|_{*},
$$

where $f_{j}$ is the scattering amplitude related to potential $v_{j}, j=1,2$, defined as

$$
f_{j}\left(\lambda, \lambda^{\prime}\right)=\left(\frac{1}{2 \pi}\right)^{2} \int_{\mathbb{C}} \exp \left[-\frac{i \sqrt{E}}{2}\left(\lambda^{\prime} \bar{z}+\frac{z}{\lambda^{\prime}}\right)\right] v_{j}(z) \varphi_{j}^{+}(z, \lambda) d \operatorname{Re} z d \operatorname{Im} z .
$$

Here we used the change of variables in Section 2 , and $\varphi_{j}^{+}(x, k)=\psi_{k /|k|}(x, k)$, where $\psi_{\gamma}(x, k)$ was defined in (2.5). The following identity holds:

$$
\begin{aligned}
& f_{2}\left(\lambda, \lambda^{\prime}\right)-f_{1}\left(\lambda, \lambda^{\prime}\right) \\
& \quad=\left(\frac{1}{2 \pi}\right)^{2} \int_{\partial D} \varphi_{1}^{+}\left(x,-k\left(\lambda^{\prime}\right)\right)\left(\Phi_{2}(E)-\Phi_{1}(E)\right) \varphi_{2}^{+}(x, k(\lambda)) d x,
\end{aligned}
$$


HÖLDER-LOGARITHMIC STABILITY ESTIMATE IN 2D AT POSITIVE ENERGY 11 for $|\lambda|=\left|\lambda^{\prime}\right|=1$. This is proved in [23, Theorem 1]. We then obtain

$$
\begin{aligned}
& \left|f_{2}\left(\lambda, \lambda^{\prime}\right)-f_{1}\left(\lambda, \lambda^{\prime}\right)\right| \\
& \quad \leq \frac{1}{(2 \pi)^{2}}\left\|\varphi_{1}^{+}(\cdot, k)\right\|_{L^{\infty}(\partial D)}\left\|\Phi_{2}(E)-\Phi_{1}(E)\right\|\left\|_{*}\right\| \varphi_{2}^{+}(\cdot, k) \|_{L^{\infty}(\partial D)},
\end{aligned}
$$

where $|\lambda|=\left|\lambda^{\prime}\right|=1$ and so $k \in \mathbb{R}^{2}, k^{2}=E$. From Lemma 2.1 we get

$$
\left\|\varphi_{j}^{+}(\cdot, k)\right\|_{L^{\infty}(\partial D)}=\left\|\mu_{k /|k|}(\cdot, k)\right\|_{L^{\infty}(\partial D)} \leq c(D, N, m),
$$

for $j=1,2$, since $k \in \mathbb{R}^{2}, k^{2}=E$. This, combined with (3.8), gives (3.7).

It is now useful to recall the following integral equations which relate $f_{j}$ with $h_{ \pm}^{j}($ see [10, 21]):

$$
\begin{gathered}
h_{ \pm}^{j}\left(\lambda, \lambda^{\prime}\right)-\pi i \int_{\left|\lambda^{\prime \prime}\right|=1} h_{ \pm}^{j}\left(\lambda, \lambda^{\prime \prime}\right) \theta\left[ \pm \frac{1}{i}\left(\frac{\lambda^{\prime \prime}}{\lambda}-\frac{\lambda}{\lambda^{\prime \prime}}\right)\right] \\
\times f_{j}\left(\lambda^{\prime \prime}, \lambda^{\prime}\right)\left|d \lambda^{\prime \prime}\right|=f_{j}\left(\lambda, \lambda^{\prime}\right) \quad j=1,2 .
\end{gathered}
$$

Subtracting this equation for $j=2$ and $j=1$ we obtain

$$
\left(I+P_{ \pm}^{2}\right)\left(h_{ \pm}^{2}-h_{ \pm}^{1}\right)=\left(I+Q_{ \pm}^{1}\right)\left(f_{2}-f_{1}\right)
$$

where

$$
\begin{aligned}
& \left(P_{ \pm}^{j} u\right)\left(\lambda, \lambda^{\prime}\right)=-\pi i \int_{\lambda^{\prime \prime} \in T} u\left(\lambda, \lambda^{\prime \prime}\right) \theta\left[ \pm \frac{1}{i}\left(\frac{\lambda^{\prime \prime}}{\lambda}-\frac{\lambda}{\lambda^{\prime \prime}}\right)\right] f_{j}\left(\lambda^{\prime \prime}, \lambda^{\prime}\right)\left|d \lambda^{\prime \prime}\right|,
\end{aligned}
$$

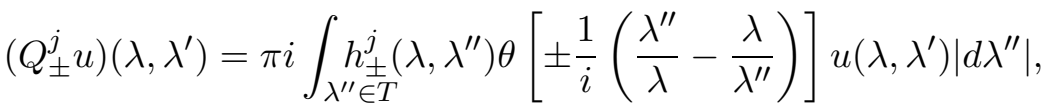

for $u \in L^{p}\left(T^{2}\right), p>1$. In [22, $\left.\S 2\right]$ it is proved that

$$
\begin{aligned}
\left|f_{j}\left(\lambda, \lambda^{\prime}\right)\right| & \leq 2\left\|\hat{v}_{j}\right\|_{\alpha, m}\left(1+E\left|\lambda-\lambda^{\prime}\right|^{2}\right)^{-m / 2}, \\
\left|h_{ \pm}^{j}\left(\lambda, \lambda^{\prime}\right)\right| & \leq 2\left\|\hat{v}_{j}\right\|_{\alpha, m}\left(1+E\left|\lambda-\lambda^{\prime}\right|^{2}\right)^{-m / 2},
\end{aligned}
$$

for $\lambda, \lambda^{\prime} \in T$ and $E \geq E_{1}=E_{1}(N, D, m)$. From these inequalities (and also inequalities (2.45) of [22]) we find that

$$
\begin{aligned}
\left\|P_{ \pm}^{j} u\right\|_{L^{2}(T \times T)} & \leq \frac{c_{1}(N, m)}{E^{1 / 4}}\|u\|_{L^{2}(T \times T)}, \\
\left\|Q_{ \pm}^{j} u\right\|_{L^{2}(T \times T)} & \leq \frac{c_{2}(N, m)}{E^{1 / 4}}\|u\|_{L^{2}(T \times T)} .
\end{aligned}
$$

Choose $E_{1}^{\prime} \geq E_{1}$ such that $\max \left(\frac{c_{1}(N, m)}{E^{\prime 1 / 4}}, \frac{c_{2}(N, m)}{E^{\prime 1 / 4}}\right) \leq \frac{1}{2}$. Then $P_{ \pm}^{j}$ is invertible on $L^{2}(T \times T)$ and from (3.10) we obtain

$$
\left\|h_{ \pm}^{2}-h_{ \pm}^{1}\right\|_{L^{2}(T \times T)} \leq c_{3}(N, D, m)\left\|f_{2}-f_{1}\right\|_{L^{2}(T \times T)},
$$


for $E \geq E_{1}^{\prime}$. It is straightforward to see that

$$
\left\|h_{\beta}^{2}-h_{\beta}^{1}\right\|_{L^{2}(T \times T)} \leq\left\|h_{+}^{2}-h_{+}^{1}\right\|_{L^{2}(T \times T)}+\left\|h_{-}^{2}-h_{-}^{1}\right\|_{L^{2}(T \times T)}, \beta=1,2,
$$

where $h_{\beta}^{j}$ are the auxiliary functions defined in (2.11) and (2.12) related to the potential $v_{j}, j=1,2$.

In order to finish the proof, it is sufficient to remark that the functions $h_{\beta}^{j}$ satisfy inequality (3.14), as well as $\rho_{j}$. For this, we will need the following lemma, which will be proved later.

Lemma 3.4. The function $\rho$, defined in (2.13) for a potential $v$ such that $\|v\|_{m, 1} \leq N$, satisfies the inequality

$$
\left|\rho\left(\lambda, \lambda^{\prime}\right)\right| \leq c(N)\left(1+E\left|\lambda-\lambda^{\prime}\right|^{2}\right)^{-m / 2},
$$

for $\lambda, \lambda^{\prime} \in T$ and $E \geq \tilde{E}_{1}=\tilde{E}_{1}(N, D, m)$.

Now we see that thanks to Lemma 3.4, equations (2.13) have the same structure of equations (3.9), and the kernels satisfy the same inequalities for $E \geq E_{2}\left(\tilde{E}_{1}, E_{1}^{\prime}\right)$. Thus we obtain directly

$$
\left\|\rho_{2}-\rho_{1}\right\|_{L^{2}(T \times T)} \leq c_{4}(N, D, m)\left\|h_{\beta}^{2}-h_{\beta}^{1}\right\|_{L^{2}(T \times T)}, \beta=1,2,
$$

for $E \geq E_{2}$.

Now inequalities (3.20), (3.18), (3.17) together with (3.7) give (3.6), which finishes the proof.

Proof of Lemma 3.4. We write the integral equation defining $\rho$, (2.13a), as follows:

$$
\left(I+H_{1}\right) \rho\left(\lambda, \lambda^{\prime}\right)=-\pi i h_{1}\left(\lambda, \lambda^{\prime}\right)
$$

where

$$
H_{1} \rho\left(\lambda, \lambda^{\prime}\right)=\pi i \int_{\left|\lambda^{\prime \prime}\right|=1} \rho\left(\lambda, \lambda^{\prime \prime}\right) \theta\left[\frac{1}{i}\left(\frac{\lambda^{\prime}}{\lambda^{\prime \prime}}-\frac{\lambda^{\prime \prime}}{\lambda^{\prime}}\right)\right] h_{1}\left(\lambda^{\prime \prime}, \lambda^{\prime}\right)\left|d \lambda^{\prime \prime}\right| .
$$

We want to prove that this equation has a unique solution in the space of complex-valued functions $g\left(\lambda, \lambda^{\prime}\right)$ defined on $T^{2}$, such that

$$
\left|g\left(\lambda, \lambda^{\prime}\right)\right| \leq c\left(1+E\left|\lambda-\lambda^{\prime}\right|^{2}\right)^{-m / 2},
$$

for some constant $c$. Let us call this function space $S$ and define $\|g\|_{S}=\inf c$ such that (3.23) is verified.

We have that $\left\|H_{1} g\right\|_{S} \leq c E^{-1 / 2}\|g\|_{S}$. Indeed, since $h_{1}$ satisfies inequality (3.14), the following estimate holds

$$
\left|H_{1} g\left(\lambda, \lambda^{\prime}\right)\right| \leq c\|g\|_{S} \int_{T} \frac{\left|d \lambda^{\prime \prime}\right|}{\left(\left(1+E\left|\lambda-\lambda^{\prime \prime}\right|^{2}\right)\left(1+E\left|\lambda^{\prime \prime}-\lambda^{\prime}\right|^{2}\right)\right)^{m / 2}} .
$$


We split the circle $T$, at fixed $\lambda, \lambda^{\prime}$ in two sets: the first contains the points $\lambda^{\prime \prime}$ that are closer to $\lambda$ than to $\lambda^{\prime}$, i.e. $\left|\lambda^{\prime \prime}-\lambda\right| \leq\left|\lambda^{\prime \prime}-\lambda^{\prime}\right|$ and the second is the complement. For $\lambda^{\prime \prime}$ in the first set we have that $\left|\lambda^{\prime \prime}-\lambda^{\prime}\right| \geq \frac{1}{2}\left|\lambda^{\prime}-\lambda\right|$ while for $\lambda^{\prime \prime}$ in the second $\left|\lambda^{\prime \prime}-\lambda\right| \geq \frac{1}{2}\left|\lambda^{\prime}-\lambda\right|$. Thus we obtain

$$
\left|H_{1} g\left(\lambda, \lambda^{\prime}\right)\right| \leq c \frac{\|g\|_{S}}{\left(2+E\left|\lambda-\lambda^{\prime}\right|^{2}\right)^{m / 2}} \int_{T} \frac{\left|d \lambda^{\prime \prime}\right|}{\left(1+E\left|\lambda^{\prime \prime}-\tilde{\lambda}\right|^{2}\right)^{m / 2}},
$$

where $\tilde{\lambda}$ is some point in $T$. Using inequality (4.28) we obtain the estimate for $H_{1}$. Then for sufficiently large $E$, equation (3.21) has a unique solution in $S$ by iteration. This finishes the proof of Lemma 3.4 .

In the following proposition we prove that the map $\Phi(E) \rightarrow r(\lambda)$ is logarithmic stable.

Proposition 3.5. Let $E$ be such that $E \geq E_{3}=\max \left((2 R)^{2}, E_{0}\right)$, where $R$ is defined in Lemma 2.2 and $E_{0}$ in Remark 2.1, let $v_{1}, v_{2}$ be two potentials satisfying (1.2), (1.5), (1.7), $\Phi_{1}(E), \Phi_{2}(E)$ the corresponding Dirichlet-toNeumann operator and $r_{1}, r_{2}$ as defined in (2.16). Let $\left\|v_{k}\right\|_{m, 1} \leq N, k=1,2$. Then for every $p \geq 1$ there exists a constant $\theta=\theta(D, N, m, p)$ such that for any $0 \leq \kappa<\frac{1}{4(l+1)}$, where $l=\operatorname{diam}(D)$, and for $E \geq E_{3}$ we have

$$
\begin{gathered}
\left\|\left(|\lambda|+\frac{1}{|\lambda|}\right)\left|r_{2}-r_{1}\right|\right\|_{L^{p}(\mathbb{C})}^{\leq} \\
\theta\left[E^{-1}\left(E^{1 / 2}+\kappa \log \left(3+\delta^{-1}\right)\right)^{-(m-2)}\right. \\
\left.+\frac{\delta\left(3+\delta^{-1}\right)^{4 \kappa(l+1)}}{E^{1 / 2 p}}\right]
\end{gathered}
$$

where $\delta=\left\|\Phi_{2}(E)-\Phi_{1}(E)\right\|_{L^{\infty}(\partial D) \rightarrow L^{\infty}(\partial D)}$.

Proof. We choose $0<a_{1} \leq 1 \leq a_{2}$ to be determined and split down the left hand side of $(3.26)$ as follows:

$$
\begin{gathered}
\left\|\left(|\lambda|+\frac{1}{|\lambda|}\right)\left|r_{2}-r_{1}\right|\right\|_{L^{p}(\mathbb{C})} \leq I_{1}+I_{2}+I_{3}, \\
I_{1}=\left\|\left(|\lambda|+\frac{1}{|\lambda|}\right)\left|r_{2}-r_{1}\right|\right\|_{L^{p}\left(|\lambda|<a_{1}\right)}, \\
I_{2}=\left\|\left(|\lambda|+\frac{1}{|\lambda|}\right)\left|r_{2}-r_{1}\right|\right\|_{L^{p}\left(a_{1}<|\lambda|<a_{2}\right)}, \\
I_{3}=\left\|\left(|\lambda|+\frac{1}{|\lambda|}\right)\left|r_{2}-r_{1}\right|\right\|_{L^{p}\left(|\lambda|>a_{2}\right)} .
\end{gathered}
$$

From (3.1) and (3.2) we have

$$
\begin{aligned}
& I_{1} \leq c(N, p, m) E^{-m / 2} a_{1}^{m-2+2 / p}, \\
& I_{3} \leq c(N, p, m) E^{-m / 2} a_{2}^{-m+2 / p}
\end{aligned}
$$


Lemma 3.2 yields that $I_{2}$ can be estimated from above by

$$
c(D, N, p) \frac{\delta}{E^{1 / 2 p}}\left(e^{4(l+1) \sqrt{E}\left(\frac{1}{a_{1}}-1\right)}+e^{4(l+1) \sqrt{E}\left(a_{2}-1\right)}\right) .
$$

Here we used the fact that

$$
\begin{aligned}
\left(\frac{1}{|\lambda|}+|\lambda|\right) \frac{e^{l \sqrt{E}|| \lambda|-1 /| \lambda||}}{|\lambda|} & \leq e^{2 l \sqrt{E}|| \lambda|-1 /| \lambda||} \\
& \leq e^{4 l \sqrt{E}(1 /|\lambda|-1)} \chi_{|\lambda|<1}+e^{4 l \sqrt{E}(|\lambda|-1)} \chi_{|\lambda|>1}
\end{aligned}
$$

where $\chi_{A}$ is the characteristic function of a set $A \subset \mathbb{C}$.

Now we define, in (3.27)-(3.29),

$$
a_{2}=\frac{1}{a_{1}}=1+\frac{\kappa \log \left(3+\delta^{-1}\right)}{\sqrt{E}},
$$

for $0 \leq \kappa<\frac{1}{4(l+1)}$. Note that $a_{2} \geq 1$ and $a_{1} \leq 1$. Then we obtain, for every $p \geq 1$,

$$
I_{j} \leq c(N, p, m) E^{-1}\left(\sqrt{E}+\kappa \log \left(3+\delta^{-1}\right)\right)^{-(m-2)}, \quad j=1,3,
$$

To estimate $I_{2}$ we remark that

$$
\begin{aligned}
e^{4(l+1) \sqrt{E}\left(\frac{1}{a_{1}}-1\right)}+e^{4(l+1) \sqrt{E}\left(a_{2}-1\right)} & =2 e^{2(l+1) \kappa \log \left(3+\delta^{-1}\right)} \\
& =2\left(3+\delta^{-1}\right)^{4(l+1) \kappa} .
\end{aligned}
$$

Putting (3.32)-(3.33) together we find

$$
\begin{aligned}
\left\|\left(|\lambda|+\frac{1}{|\lambda|}\right)\left|r_{2}-r_{1}\right|\right\| \underset{L^{p}(\mathbb{C})}{\leq} & \theta_{2}\left[E^{-1}\left(E^{1 / 2}+\kappa \log \left(3+\delta^{-1}\right)\right)^{-(m-2)}\right. \\
& \left.+\frac{\delta\left(3+\delta^{-1}\right)^{4(l+1) \kappa}}{E^{1 / 2 p}}\right]
\end{aligned}
$$

which is estimate (3.26).

Remark 3.1. In the following sections we will often implicitly use the basic fact that

$$
\left\|r_{2}-r_{1}\right\|_{L^{p}(\mathbb{C})} \leq\left\|\left(|\lambda|+\frac{1}{|\lambda|}\right)\left|r_{2}-r_{1}\right|\right\|_{L^{p}(\mathbb{C})}
$$

\section{Estimates for the NON-LOCAl Riemann-Hilbert PROBlem}

We begin with an explicit formula relating a potential $v$, satisfying the assumption of Theorem 1.1, with its associated functions $r(\lambda)$ and $\rho\left(\lambda, \lambda^{\prime}\right)$. This procedure allows us to explicitly solve the non-local Riemann-Hilbert problem presented in Section 2 (see (2.14) $-(2.18)$ ). 
The starting point is formula (2.21):

$$
\begin{array}{r}
v(z)=2 i \sqrt{E} \frac{\partial}{\partial z} \mu_{-1}(z), \\
\mu_{-1}(z)=\lim _{\lambda \rightarrow \infty} \lambda(\mu(z, \lambda)-1) .
\end{array}
$$

We follow the scheme of [21, Theorem 6.1] in order to make $\mu$ explicit. In the following equations we omit the variable $z$ in the functions $\mu, e, K, \Omega, X$ for simplicity's sake. We have

$$
\begin{aligned}
\mu(\lambda)=e(\lambda)+\frac{1}{2 \pi i} \int_{|\zeta|=1} \Omega_{1}(\lambda, \zeta) K(\zeta) d \zeta-\Omega_{2}(\lambda, \zeta) \overline{K(\zeta)} d \bar{\zeta} \\
e(\lambda)=1-\frac{1}{\pi} \int_{\mathbb{C}} \frac{r(\zeta, z) \overline{e(\zeta)}}{\zeta-\lambda} d \operatorname{Re} \zeta d \operatorname{Im} \zeta \\
\Omega_{1}(\lambda, \zeta)=X_{1}(\lambda, \zeta)+i X_{2}(\lambda, \zeta), \\
\Omega_{2}(\lambda, \zeta)=X_{1}(\lambda, \zeta)-i X_{2}(\lambda, \zeta), \\
X_{1}(\lambda, \zeta)+\frac{1}{\pi} \int_{\mathbb{C}} \frac{r(\eta, z) \overline{X_{1}(\eta, \zeta)}}{\eta-\lambda} d \operatorname{Re} \eta d \operatorname{Im} \eta=\frac{1}{2(\zeta-\lambda)}, \\
X_{2}(\lambda, \zeta)+\frac{1}{\pi} \int_{\mathbb{C}} \frac{r(\eta, z) \overline{X_{2}(\eta, \zeta)}}{\eta-\lambda} d \operatorname{Re} \eta d \operatorname{Im} \eta=\frac{1}{2 i(\zeta-\lambda)}, \\
K(\lambda)=\mu_{+}(\lambda)-\mu-(\lambda) \\
=\int_{\left|\lambda^{\prime}\right|=1} \rho\left(\lambda, \lambda^{\prime}, z\right)\left[e\left(\lambda^{\prime}\right)\right. \\
+\frac{1}{2 \pi i} \int_{|\zeta|=1} \Omega_{1}\left(\lambda^{\prime}(1+0), \zeta\right) K(\zeta) d \zeta \\
\left.+\Omega_{2}\left(\lambda^{\prime}, \zeta\right) \overline{K(\zeta)} d \bar{\zeta}\right]\left|d \lambda^{\prime}\right| .
\end{aligned}
$$

Let, for $p \geq 1, \nu \geq 0, L_{\nu}^{p}(\mathbb{C})$ be the function space

$$
\left\{f:\left.\mathbb{C} \rightarrow \mathbb{C}\left|f(z) \in L^{p}(|z| \leq 1),\right| z\right|^{-\nu} f\left(\frac{1}{|z|}\right) \in L^{p}(|z| \leq 1)\right\},
$$

with the corresponding norm $\|f\|_{L_{\nu}^{p}(\mathbb{C})}=\|f\|_{L^{p}(|z| \leq 1)}+\left\||z|^{-\nu} f(1 /|z|)\right\|_{L^{p}(|z| \leq 1)}$. From Lemma 2.2 we have that $r_{j} \in L_{\nu}^{p}(\mathbb{C})$ for all $\nu<m$. Then, from results of [31], equations (4.4), (4.7) and (4.8) are uniquely solved in $L_{0}^{q}(\mathbb{C})$, $p /(p-1) \leq q<2$, and $e(\lambda)$ is continuous on $\mathbb{C}$.

Then we can write

$$
\begin{aligned}
& \mu_{-1}(z)=\frac{1}{\pi} \int_{\mathbb{C}} r(\zeta, z) \overline{e(\zeta)} d \operatorname{Re} \zeta d \operatorname{Im} \zeta \\
& +\frac{1}{2 \pi i} \int_{|\zeta|=1} K(\zeta)\left[-1+\frac{1}{\pi} \int_{\mathbb{C}} r(\lambda, z) \overline{\Omega_{2}(\lambda, \zeta)} d \operatorname{Re} \lambda d \operatorname{Im} \lambda\right] d \zeta \\
& \quad-\frac{1}{2 \pi i} \int_{|\zeta|=1} \overline{K(\zeta)}\left[\frac{1}{\pi} \int_{\mathbb{C}} r(\lambda, z) \overline{\Omega_{1}(\lambda, \zeta)} d \operatorname{Re} \lambda d \operatorname{Im} \lambda\right] d \bar{\zeta}
\end{aligned}
$$


where $\mu_{-1}$ was defined in (4.2). Indeed, by Lebesgue's dominated convergence (using Lemma 2.2), we can calculate the following limits:

$$
\begin{aligned}
& \lim _{\lambda \rightarrow \infty} \lambda(e(\lambda)-1)=\frac{1}{\pi} \int_{\mathbb{C}} r(\zeta, z) \overline{e(\zeta)} d \operatorname{Re} \zeta d \operatorname{Im} \zeta \\
& \lim _{\lambda \rightarrow \infty} \lambda X_{1}(\lambda, \zeta)=-\frac{1}{2}+\frac{1}{\pi} \int_{\mathbb{C}} r(\eta, z) \overline{X_{1}(\eta, \zeta)} d \operatorname{Re} \eta d \operatorname{Im} \eta \\
& \lim _{\lambda \rightarrow \infty} \lambda X_{2}(\lambda, \zeta)=-\frac{1}{2 i}+\frac{1}{\pi} \int_{\mathbb{C}} r(\eta, z) \overline{X_{2}(\eta, \zeta)} d \operatorname{Re} \eta d \operatorname{Im} \eta
\end{aligned}
$$

Then, in connection with (4.1), we need to take the derivative of (4.11) with respect to $\partial / \partial z=\partial_{z}$. We find:

$$
\partial_{z} \mu_{-1}=A+B+C
$$

where

$$
\begin{aligned}
A=\frac{1}{\pi} \int_{\mathbb{C}} r(\zeta, z)\left[-\frac{i}{2} \sqrt{E}\left(\frac{1}{\zeta}+\bar{\zeta}\right) \overline{e(\zeta)}+\overline{\partial_{\bar{z}} e(\zeta)}\right] d \operatorname{Re} \zeta d \operatorname{Im} \zeta \\
B=\frac{1}{2 \pi i} \int_{|\zeta|=1}\left\{\partial_{z} K(\zeta)\left[-1+\frac{1}{\pi} \int_{\mathbb{C}} r(\lambda, z) \overline{\Omega_{2}(\lambda, \zeta)} d \operatorname{Re} \lambda d \operatorname{Im} \lambda\right]\right. \\
\quad+\frac{K(\zeta)}{\pi} \int_{\mathbb{C}}\left[r ( \lambda , z ) \left(-\frac{i}{2} \sqrt{E}\left(\frac{1}{\lambda}+\bar{\lambda}\right) \overline{\Omega_{2}(\lambda, \zeta)}\right.\right. \\
\left.\left.\left.\quad+\overline{\partial_{\bar{z}} \Omega_{2}(\lambda, \zeta)}\right)\right] d \operatorname{Re} \lambda d \operatorname{Im} \lambda\right\} d \zeta \\
C=-\frac{1}{2 \pi i} \int_{|\zeta|=1}\left\{\overline{\partial_{\bar{z}} K(\zeta)}\left[\frac{1}{\pi} \int_{\mathbb{C}} r(\lambda, z) \overline{\Omega_{1}(\lambda, \zeta)} d \operatorname{Re} \lambda d \operatorname{Im} \lambda\right]\right. \\
\quad+\frac{\overline{K(\zeta)}}{\pi} \int_{\mathbb{C}}\left[r ( \lambda , z ) \left(-\frac{i}{2} \sqrt{E}\left(\frac{1}{\lambda}+\bar{\lambda}\right) \overline{\Omega_{1}(\lambda, \zeta)}\right.\right. \\
\quad+\frac{\left.\left.\left.\overline{\partial_{\bar{z}} \Omega_{1}(\lambda, \zeta)}\right)\right] d \operatorname{Re} \lambda d \operatorname{Im} \lambda\right\} d \bar{\zeta} .}{}
\end{aligned}
$$

Now let $v_{1}, v_{2}$ be two potential satisfying the assumptions of Theorem 1.1. Let $\mu_{-1}^{j}, r_{j}, \rho_{j}, e_{j}, K_{j}, \Omega_{1}^{j}, \Omega_{2}^{j}, X_{1}^{j}, X_{2}^{j}, A_{j}, B_{j}, C_{j}$ the above-defined functions corresponding to $v_{j}$, for $j=1,2$. Then

$$
v_{2}(z)-v_{1}(z)=2 i \sqrt{E}\left(A_{2}-A_{1}+B_{2}-B_{1}+C_{2}-C_{1}\right) .
$$

In order to estimate $A_{2}-A_{1}, B_{2}-B_{1}$ and $C_{2}-C_{1}$ we will need the following two propositions.

Proposition 4.1. Let $v_{j}, j=1,2$, be two potential satisfying the assumptions of Theorem 1.1. Then we have, for every $a>1, E \geq E_{3}(N, m)$ and 
HÖLDER-LOGARITHMIC STABILITY ESTIMATE IN 2D AT POSITIVE ENERGY 17 $1<s<2<s^{\prime}<+\infty$,

$$
\begin{aligned}
\left\|K_{2}-K_{1}\right\|_{L^{2}(T)} \leq & c(N, m)\left[\left\|\rho_{2}-\rho_{1}\right\|_{L^{2}\left(T^{2}\right)}+\frac{\left\|r_{2}-r_{1}\right\|_{L^{s, s^{\prime}}(\mathbb{C})}}{\sqrt{E}}\right. \\
& \left.+\frac{1}{E}\left(\delta r_{a}+\frac{a}{a-1}\left\|r_{2}-r_{1}\right\|_{L^{s, s^{\prime}}\left(\mathbb{C} \backslash D_{a}\right)}\right)\right],
\end{aligned}
$$

where $\|\cdot\|_{L^{s, s^{\prime}}}=\|\cdot\|_{L^{s}}+\|\cdot\|_{L^{s^{\prime}}}, T$ is the unit circle and

$$
\begin{gathered}
D_{a}=\{\lambda \in \mathbb{C}|1 / a<| \lambda \mid<a\}, \\
\delta r_{a}=\sup _{\lambda \in D_{a}}\left|r_{2}(\lambda, z)-r_{1}(\lambda, z)\right| .
\end{gathered}
$$

Proposition 4.2. Let $v_{j}, j=1,2$, be two potential satisfying the assumptions of Theorem 1.1. Then we have, for every $a>1, E \geq E_{4}(N, m)$ and for some $1<s<2<s^{\prime}<+\infty$,

$$
\begin{aligned}
\| & \nabla K_{2}-\nabla K_{1} \|_{L^{2}(T)} \leq c(N, m)\left[E^{1 / 2}\left\|\rho_{2}-\rho_{1}\right\|_{L^{2}\left(T^{2}\right)}\right. \\
& +\left\|\left(\frac{1}{|\lambda|}+|\lambda|\right)\left(r_{2}-r_{1}\right)\right\|_{L^{s, s^{\prime}}(\mathbb{C})} \\
& \left.+E^{-1}\left(\delta r_{a}^{\prime}+\frac{a}{a-1}\left\|\left(\frac{1}{|\lambda|}+|\lambda|\right)\left(r_{2}-r_{1}\right)\right\|_{L^{s, s^{\prime}}\left(\mathbb{C} \backslash D_{a}\right)}\right)\right]
\end{aligned}
$$

where $\nabla$ is taken with respect to $z,\|\cdot\|_{L^{s, s^{\prime}}}=\|\cdot\|_{L^{s}}+\|\cdot\|_{L^{s^{\prime}}}, D_{a}$ is defined in (4.17) and

$$
\delta r_{a}^{\prime}=\sup _{D_{a}}\left(\frac{1}{|\lambda|}+|\lambda|\right)\left|\left(r_{2}-r_{1}\right)(\lambda)\right| .
$$

Proof of Proposition 4.1. We rewrite integral equation (4.9) for $K_{j}$ as follows:

$$
\left(I-\Theta_{j}\right) K_{j}(\lambda)=\int_{\left|\lambda^{\prime}\right|=1} \rho_{j}\left(\lambda, \lambda^{\prime}, z\right) e_{j}\left(\lambda^{\prime}\right)\left|d \lambda^{\prime}\right|
$$

where

$$
\begin{aligned}
\Theta_{j} f(\lambda)= & \frac{1}{2 \pi i} \int_{\left|\lambda^{\prime}\right|=1} \rho_{j}\left(\lambda, \lambda^{\prime}, z\right)\left[\int_{|\zeta|=1} \Omega_{1}^{j}\left(\lambda^{\prime}(1+0), \zeta\right) f(\zeta) d \zeta\right. \\
& \left.+\Omega_{2}^{j}\left(\lambda^{\prime}, \zeta\right) \overline{f(\zeta)} d \bar{\zeta}\right]\left|d \lambda^{\prime}\right|, \quad j=1,2,
\end{aligned}
$$

for $f \in L^{2}(T)$. Subtracting equation (4.21) for $j=2$ and $j=1$ gives

$$
\begin{aligned}
& \left(I-\Theta_{2}\right)\left(K_{2}-K_{1}\right)(\lambda)=\left(\Theta_{2}-\Theta_{1}\right) K_{1}(\lambda) \\
& \quad+\int_{\left|\lambda^{\prime}\right|=1}\left(\rho_{2}-\rho_{1}\right)\left(\lambda, \lambda^{\prime}, z\right) e_{2}\left(\lambda^{\prime}\right)+\rho_{1}\left(\lambda, \lambda^{\prime}, z\right)\left(e_{2}-e_{1}\right)\left(\lambda^{\prime}\right)\left|d \lambda^{\prime}\right| .
\end{aligned}
$$


We will now use some results of [21] and [31, Ch. III]. Let $L_{\nu}^{p}(\mathbb{C})$ the set defined in (4.10). From Lemma 2.2 we have that $r_{j} \in L_{\nu}^{p}(\mathbb{C})$ for all $\nu<m$. In particular $\left\|r_{j}\right\|_{L_{2}^{p}(\mathbb{C})} \leq c(N, p) E^{-m / 2}$, for $m>2, p \geq 1$. Then we following estimates holds

$$
\begin{aligned}
\left|\Omega_{1}^{j}(\lambda, \zeta)-\frac{1}{\zeta-\lambda}\right| & \leq c(N, m, p) E^{-m / 2} \frac{1}{|\zeta-\lambda|^{2 / p}}, \\
\left|\Omega_{2}^{j}(\lambda, \zeta)\right| & \leq c(N, m, p) E^{-m / 2} \frac{1}{|\zeta-\lambda|^{2 / p}},
\end{aligned}
$$

since $\left\|r_{j}\right\|_{L_{2}^{p}(\mathbb{C})} \leq c(N, m, p) E^{-m / 2}, j=1,2$. These estimates are proved in [31, Ch. III, §8]. We also recall the following classical inequality:

$$
\begin{aligned}
& \left\|C_{ \pm} u\right\|_{L^{p}(T)} \leq c(p)\|u\|_{L^{p}(T)}, \quad 1<p<+\infty \\
& \left(C_{ \pm} u\right)(\lambda)=\frac{1}{2 \pi i} \int_{T} \frac{u(\zeta)}{\zeta-\lambda(1 \mp 0)} d \zeta .
\end{aligned}
$$

Then we have, for $p>4$,

$$
\begin{aligned}
\left\|\Theta_{j} f\right\|_{L^{2}(T)} & \leq c\left(r_{0}, p\right) \sup _{\lambda \in T}\left\|\rho_{j}(\lambda, \cdot, z)\right\|_{L^{1}(T)}\left(1+\left\|1 /|\cdot-\lambda|^{2 / p}\right\|_{L^{2}(\mathbb{C})}\right)\|f\|_{L^{2}(T)} \\
& \leq \frac{c\left(r_{o}, p\right)}{\sqrt{E}}\|f\|_{L^{2}(T)},
\end{aligned}
$$

where we used the fact that $\rho_{j}$ satisfies inequality (3.14) and

$$
\int_{T}\left(1+E\left|\lambda-\lambda^{\prime}\right|^{2}\right)^{-m / 2}\left|d \lambda^{\prime}\right| \leq c E^{-1 / 2} .
$$

Then for $E \geq E_{4}\left(r_{0}, m\right)$ we can solve equation (4.23) by iteration in $L^{2}(T)$ and find

$$
\begin{aligned}
\left\|K_{2}-K_{1}\right\|_{L^{2}(T)} \leq & c(N, m)\left(\left\|\left(\Theta_{2}-\Theta_{1}\right) K_{1}\right\|_{L^{2}(T)}\right. \\
& \left.+\left\|\rho_{2}-\rho_{1}\right\|_{L^{2}\left(T^{2}\right)}+\frac{\left\|e_{2}-e_{1}\right\|_{L^{\infty}(T)}}{\sqrt{E}}\right),
\end{aligned}
$$

where we used the $L^{\infty}(T)$-boundedness of $e_{2}$ (which follows from considerations at the beginning of this section). We have that

$$
\left\|e_{2}-e_{1}\right\|_{L^{\infty}(T)} \leq c(N, m)\left(\left\|r_{2}-r_{1}\right\|_{L^{s}(\mathbb{C})}+\left\|r_{2}-r_{1}\right\|_{L^{s^{\prime}}(\mathbb{C})}\right),
$$

for $1<s<2<s^{\prime}<+\infty$. Indeed, this follows from the integral equation $e_{2}(\lambda)-e_{1}(\lambda)=-\frac{1}{\pi} \int_{\mathbb{C}} \frac{\left(r_{2}-r_{1}\right)(\zeta, z) \overline{e_{2}(\zeta)}}{\zeta-\lambda}+\frac{r_{2}(\zeta, z) \overline{\left(e_{2}-e_{1}\right)(\zeta)}}{\zeta-\lambda} d \operatorname{Re} \zeta d \operatorname{Im} \zeta$, which is a consequence of (4.4), from Hölder's inequality, Lemma 2.2, estimate (5.5) and the $L^{\infty}$-boundedness of $e_{j}(\lambda)$ (see the beginning of this section and Section 5 for more details). 
The first term of the right hand side of (4.29), $\left(\Theta_{2}-\Theta_{1}\right) K_{1}$, can be written as

$$
\begin{aligned}
\left(\Theta_{2}-\Theta_{1}\right) K_{1}= & \frac{1}{2 \pi i} \int_{T}\left(\rho_{2}-\rho_{1}\right) \int_{T} \Omega_{1}^{2} K_{1} d \zeta+\Omega_{2}^{2} \overline{K_{1}} d \bar{\zeta}\left|d \lambda^{\prime}\right| \\
& +\frac{1}{2 \pi i} \int_{T} \rho_{1} \int_{T}\left(\Omega_{1}^{2}-\Omega_{1}^{1}\right) K_{1} d \zeta+\left(\Omega_{2}^{2}-\Omega_{2}^{1}\right) \overline{K_{1}} d \bar{\zeta}\left|d \lambda^{\prime}\right|,
\end{aligned}
$$

where we dropped the dependence on every variable for simplicity's sake. We obtain

$$
\begin{aligned}
& \left\|\left(\Theta_{2}-\Theta_{1}\right) K_{1}\right\|_{L^{2}(T)} \leq c(N, m) E^{-1 / 2}\left(\left\|\rho_{2}-\rho_{1}\right\|_{L^{2}\left(T^{2}\right)}\right. \\
& \left.\quad+E^{-1 / 2} \sup _{\lambda \in T}\left(\left\|\left(\Omega_{1}^{2}-\Omega_{1}^{1}\right)(\lambda, \cdot)\right\|_{L^{2}(T)}+\left\|\left(\Omega_{2}^{2}-\Omega_{2}^{1}\right)(\lambda, \cdot)\right\|_{L^{2}(T)}\right)\right),
\end{aligned}
$$

where we used the fact that

$$
\left\|K_{j}\right\|_{L^{\infty}(T)} \leq c(N, m) E^{-1 / 2}
$$

which follows from the first equality in (4.9), equation (2.17), inequality (3.14) for $\rho_{j}$, Lemma 2.1 and estimate (4.28).

We now need to estimate the difference of $\Omega_{k}^{j}$. The $X_{k}^{j}$ satisfy

$$
\begin{aligned}
\partial_{\bar{\lambda}}\left(X_{k}^{2}-X_{k}^{1}\right)(\lambda, \zeta)= & r_{1}(\lambda, z) \overline{\left(X_{k}^{2}-X_{k}^{1}\right)(\lambda, \zeta)} \\
& +\left(r_{2}-r_{1}\right)(\lambda, z) \overline{X_{k}^{2}}(\lambda, \zeta)
\end{aligned}
$$

for $k=1,2$. Note that the last equation holds over all the complex plane, since $X_{k}^{2}-X_{k}^{1}$ has no singularity. Moreover $\left(X_{k}^{2}-X_{k}^{1}\right)(\cdot, \zeta) \in L^{p}(\mathbb{C})$, for every $p>2$, thanks to properties of the integral operator in (4.7), (4.8), summarized in Section 5 (see (5.2) for instance). Then we may define, following [31,

$$
\begin{aligned}
w_{k}(\lambda, \zeta)= & \partial_{\bar{\lambda}}^{-1}\left(r_{1}(\cdot, z) \frac{\overline{\left(X_{k}^{2}-X_{k}^{1}\right)}(\cdot, \zeta)}{\left(X_{k}^{2}-X_{k}^{1}\right)(\cdot, \zeta)}\right)(\lambda), \\
& \partial_{\bar{\lambda}}^{-1} f(\lambda)=-\frac{1}{\pi} \int_{\mathbb{C}} \frac{f(\eta)}{\eta-\lambda} d \operatorname{Re} \eta d \operatorname{Im} \eta
\end{aligned}
$$

We have that $\left\|w_{k}\right\|_{L^{\infty}(\mathbb{C})} \leq c\left(\left\|r_{1}\right\|_{L^{s}(\mathbb{C})}+\left\|r_{1}\right\|_{L^{s^{\prime}(\mathbb{C})}}\right)$, for any fixed $1<s<$ $2<s^{\prime}<+\infty$. Thus we have the following representation formula

$$
X_{k}^{2}-X_{k}^{1}=e^{w_{k}} \partial_{\bar{\lambda}}^{-1}\left(e^{-w_{k}}\left(r_{2}-r_{1}\right) \overline{X_{k}^{2}}\right)
$$


which yields

$$
\begin{aligned}
& \left|\left(X_{k}^{2}-X_{k}^{1}\right)(\lambda, \zeta)\right| \leq c(N, m) \int_{\mathbb{C}} \frac{\left|\left(r_{2}-r_{1}\right)(\eta, z)\right|\left|X_{k}^{2}(\eta, \zeta)\right|}{|\eta-\lambda|} d \operatorname{Re} \eta d \operatorname{Im} \eta \\
& \quad \leq c(N, m) \int_{\mathbb{C}}\left(\frac{\left|\left(r_{2}-r_{1}\right)(\eta, z)\right|}{|\eta-\lambda||\zeta-\eta|}+\frac{\left|\left(r_{2}-r_{1}\right)(\eta, z)\right|}{|\eta-\lambda||\zeta-\eta|^{2 / p}}\right) d \operatorname{Re} \eta d \operatorname{Im} \eta,
\end{aligned}
$$

for $\lambda, \zeta \in T$, where we used estimates (4.24) and (4.25). Now take $a>1$ and define

$$
\begin{gathered}
D_{a}=\{\lambda \in \mathbb{C}|1 / a<| \lambda \mid<a\}, \\
\delta r_{a}=\sup _{\lambda \in D_{a}}\left|r_{2}(\lambda, z)-r_{1}(\lambda, z)\right| .
\end{gathered}
$$

We then estimate the last integral in (4.37) on $D_{a}$ and on $\mathbb{C} \backslash D_{a}$, like in [21, Theorem 6.1], and we obtain, using Hölder's inequality,

$\left|\left(X_{k}^{2}-X_{k}^{1}\right)(\lambda, \zeta)\right| \leq c(N, m)\left(\delta r_{a}|\log | \lambda-\zeta\left\|+\frac{a}{a-1}\right\| r_{2}-r_{1} \|_{L^{s, s^{\prime}}\left(\mathbb{C} \backslash D_{a}\right)}\right)$,

for $\lambda, \zeta \in T, 1<s<2<s^{\prime}<+\infty$, since $\frac{a}{a-1}=\max \left(\frac{1}{a-1}, \frac{1}{1-1 / a}\right)$. We then get

$\sup _{\lambda \in T}\left\|\Omega_{k}^{2}(\lambda, \cdot)-\Omega_{k}^{1}(\lambda, \cdot)\right\|_{L^{2}(T)} \leq c(N, m)\left(\delta r_{a}+\frac{a}{a-1}\left\|r_{2}-r_{1}\right\|_{L^{s, s^{\prime}}\left(\mathbb{C} \backslash D_{a}\right)}\right)$.

First this yields

$$
\begin{gathered}
\left\|\left(\Theta_{2}-\Theta_{1}\right) K_{1}\right\|_{L^{2}(T)} \leq c(N, m) E^{-1 / 2}\left[\left\|\rho_{2}-\rho_{1}\right\|_{L^{2}\left(T^{2}\right)}\right. \\
\left.+E^{-1 / 2}\left(\delta r_{a}+\frac{a}{a-1}\left\|r_{2}-r_{1}\right\|_{L^{s, s^{\prime}}\left(\mathbb{C} \backslash D_{a}\right)}\right)\right]
\end{gathered}
$$

and finally

$$
\begin{aligned}
\left\|K_{2}-K_{1}\right\|_{L^{2}(T)} \leq & c(N, m)\left[\left\|\rho_{2}-\rho_{1}\right\|_{L^{2}\left(T^{2}\right)}+\frac{\left\|r_{2}-r_{1}\right\|_{L^{s, s^{\prime}}(\mathbb{C})}}{\sqrt{E}}\right. \\
& \left.+\frac{1}{E}\left(\delta r_{a}+\frac{a}{a-1}\left\|r_{2}-r_{1}\right\|_{L^{s, s^{\prime}}\left(\mathbb{C} \backslash D_{a}\right)}\right)\right],
\end{aligned}
$$

which ends the proof of Proposition 4.1

Proof of Proposition 4.2. We derive integral equation (4.9) for $K_{j}$ with respect to $\partial_{z}$ and $\partial_{\bar{z}}$ and we obtain two coupled integral equations for $\partial_{z} K_{j}$ and $\partial_{\bar{z}} K_{j}$. Thus we define

$$
K_{j}^{ \pm}=\partial_{z} K_{j} \pm \partial_{\bar{z}} K_{j}, \quad j=1,2
$$



which satisfy

$$
\begin{aligned}
& \left(I-\Theta_{j}^{ \pm}\right) K_{j}^{ \pm}(\lambda)=\int_{\left|\lambda^{\prime}\right|=1} \rho_{j}^{ \pm}\left(\lambda, \lambda^{\prime}, z\right)\left[e_{j}\left(\lambda^{\prime}\right)\right. \\
& \left.\quad+\frac{1}{2 \pi i} \int_{|\zeta|=1} \Omega_{1}^{j}\left(\lambda^{\prime}(1+0), \zeta\right) K_{j}(\zeta) d \zeta+\Omega_{2}^{j}\left(\lambda^{\prime}, \zeta\right) \overline{K_{j}(\zeta)} d \bar{\zeta}\right]\left|d \lambda^{\prime}\right| \\
& \quad \int_{\left|\lambda^{\prime}\right|=1} \rho_{j}\left(\lambda, \lambda^{\prime}, z\right)\left[e_{j}^{ \pm}\left(\lambda^{\prime}\right)+\frac{1}{2 \pi i} \int_{|\zeta|=1} \Omega_{1}^{ \pm, j}\left(\lambda^{\prime}(1+0), \zeta\right) K_{j}(\zeta) d \zeta\right. \\
& \left.\quad+\Omega_{2}^{ \pm, j}\left(\lambda^{\prime}, \zeta\right) \overline{K_{j}(\zeta)} d \bar{\zeta}\right]\left|d \lambda^{\prime}\right|, \quad j=1,2,
\end{aligned}
$$

where

$$
\begin{aligned}
\Theta_{j}^{ \pm} f(\lambda)= & \frac{1}{2 \pi i} \int_{\left|\lambda^{\prime}\right|=1} \rho_{j}\left(\lambda, \lambda^{\prime}, z\right)\left[\int_{|\zeta|=1} \Omega_{1}^{j}\left(\lambda^{\prime}(1+0), \zeta\right) f(\zeta) d \zeta\right. \\
& \left. \pm \Omega_{2}^{j}\left(\lambda^{\prime}, \zeta\right) \overline{f(\zeta)} d \bar{\zeta}\right]\left|d \lambda^{\prime}\right| \\
\rho_{j}^{ \pm}= & \partial_{z} \rho_{j} \pm \partial_{\bar{z}} \rho_{j}, \quad e_{j}^{ \pm}=\partial_{z} e_{j} \pm \partial_{\bar{z}} e_{j} \\
\Omega_{k}^{ \pm, j}= & \partial_{z} \Omega_{k}^{j} \pm \partial_{\bar{z}} \Omega_{k}^{j},
\end{aligned}
$$

for $j=1,2, k=1,2$. Integral equations (4.44) are obtained by adding and subtracting the two above-mentioned coupled integral equations for $\partial_{z} K_{j}$ and $\partial_{\bar{z}} K_{j}$ (which are obtained from (4.9)).

We then subtract (4.44) for $j=1$ from $j=2$ and we get

$$
\begin{aligned}
(I & \left.-\Theta_{2}^{ \pm}\right)\left(K_{2}^{ \pm}-K_{1}^{ \pm}\right)=\left(\Theta_{2}^{ \pm}-\Theta_{1}^{ \pm}\right) K_{1}^{ \pm} \\
& +\int_{\left|\lambda^{\prime}\right|=1}\left(\rho_{2}^{ \pm}-\rho_{1}^{ \pm}\right)\left[e_{2}+\frac{1}{2 \pi i} \int_{|\zeta|=1} \Omega_{1}^{2} K_{2} d \zeta+\Omega_{2}^{2} \overline{K_{2}} d \bar{\zeta}\right]\left|d \lambda^{\prime}\right| \\
& +\int_{\left|\lambda^{\prime}\right|=1} \rho_{1}^{ \pm}\left[e_{2}-e_{1}+\frac{1}{2 \pi i} \int_{|\zeta|=1}\left(\left(\Omega_{1}^{2}-\Omega_{1}^{1}\right) K_{2}+\Omega_{1}^{1}\left(K_{2}-K_{1}\right)\right) d \zeta\right. \\
& \left.+\left(\left(\Omega_{2}^{2}-\Omega_{2}^{1}\right) \bar{K}_{2}+\Omega_{2}^{1}\left(\bar{K}_{2}-\bar{K}_{1}\right)\right) d \bar{\zeta}\right]\left|d \lambda^{\prime}\right| \\
& +\int_{\left|\lambda^{\prime}\right|=1}\left(\rho_{2}-\rho_{1}\right)\left[e_{2}^{ \pm}+\frac{1}{2 \pi i} \int_{|\zeta|=1} \Omega_{1}^{ \pm, 2} K_{2} d \zeta+\Omega_{2}^{ \pm, 2} \bar{K}_{2} d \bar{\zeta}\right]\left|d \lambda^{\prime}\right| \\
& +\int_{\left|\lambda^{\prime}\right|=1} \rho_{1}\left[e_{2}^{ \pm}-e_{1}^{ \pm}+\frac{1}{2 \pi i} \int_{|\zeta|=1}\left(\left(\Omega_{1}^{ \pm, 2}-\Omega_{1}^{ \pm, 1}\right) K_{2}+\Omega_{1}^{ \pm, 1}\left(K_{2}-K_{1}\right)\right) d \zeta\right. \\
& \left.+\left(\left(\Omega_{2}^{ \pm, 2}-\Omega_{2}^{ \pm, 1}\right) \bar{K}_{2}+\Omega_{2}^{ \pm, 1}\left(\bar{K}_{2}-\bar{K}_{1}\right)\right) d \bar{\zeta}\right]\left|d \lambda^{\prime}\right|,
\end{aligned}
$$

where we dropped the dependence on every variable for simplicity's sake. The operator $\Theta_{j}^{ \pm}$satisfies the same estimates of operator $\Theta_{j}$. Then, for $E \geq E_{4}(N, m)$ the last equation is solvable by iteration in $L^{2}(T)$ and we 
have

$$
\begin{aligned}
& \left\|K_{2}^{ \pm}-K_{1}^{ \pm}\right\|_{L^{2}(T)} \leq c(N)\left(\left\|\left(\Theta_{2}^{ \pm}-\Theta_{1}^{ \pm}\right) K_{1}^{ \pm}\right\|_{L^{2}(T)}\right. \\
& +\left\|\rho_{2}^{ \pm}-\rho_{1}^{ \pm}\right\|_{L^{2}\left(T^{2}\right)}+\sup _{\lambda \in T}\left\|\rho_{1}^{ \pm}(\lambda, \cdot)\right\|_{L^{1}(T)}\left[\left\|e_{2}-e_{1}\right\|_{L^{\infty}(T)}\right. \\
& +\left\|K_{2}-K_{1}\right\|_{L^{2}(T)}+\frac{1}{\sqrt{E}}\left(\sup _{\lambda \in T}\left\|\left(\Omega_{1}^{2}-\Omega_{1}^{1}\right)(\lambda, \cdot)\right\|_{L^{2}(T)}\right. \\
& \left.\left.\quad+\sup _{\lambda \in T}\left\|\left(\Omega_{2}^{2}-\Omega_{2}^{1}\right)(\lambda, \cdot)\right\|_{L^{2}(T)}\right)\right] \\
& \quad+\left\|\rho_{2}-\rho_{1}\right\|_{L^{2}\left(T^{2}\right)}\left[\left\|e_{2}^{ \pm}\right\|_{L^{\infty}(T)}+\left\|\int_{|\zeta|=1} \Omega_{1}^{ \pm, 2} K_{2} d \zeta+\Omega_{2}^{ \pm, 2} \bar{K}_{2} d \bar{\zeta}\right\|_{L^{2}(T)}\right] \\
& \quad+\sup _{\lambda \in T}\left\|\rho_{1}(\lambda, \cdot)\right\|_{L^{1}(T)}\left[\left\|e_{2}^{ \pm}-e_{1}^{ \pm}\right\|_{L^{\infty}(T)}\right. \\
& +\frac{1}{2 \pi i} \| \int_{|\zeta|=1}\left(\left(\Omega_{1}^{ \pm, 2}-\Omega_{1}^{ \pm, 1}\right) K_{2}+\Omega_{1}^{ \pm, 1}\left(K_{2}-K_{1}\right)\right) d \zeta \\
& \left.\left.+\left(\left(\Omega_{2}^{ \pm, 2}-\Omega_{2}^{ \pm, 1}\right) \bar{K}_{2}+\Omega_{2}^{ \pm, 1}\left(\bar{K}_{2}-\bar{K}_{1}\right)\right) d \bar{\zeta} \|_{L^{2}(T)}\right]\right)
\end{aligned}
$$

where we used the $L^{\infty}$-boundedness of $e_{j}$ (see the beginning of this section) and estimates (4.24), (4.25) as in the proof of Proposition 4.1.

Since the kernels of $\Theta_{j}$ and $\Theta_{j}^{ \pm}$differ only by a sign, estimate (4.42) yields

$$
\begin{aligned}
& \left\|\left(\Theta_{2}^{ \pm}-\Theta_{1}^{ \pm}\right) K_{1}^{ \pm}\right\|_{L^{2}(T)} \leq c(N, m) E^{-1 / 2}\left[\left\|\rho_{2}-\rho_{1}\right\|_{L^{2}\left(T^{2}\right)}\right. \\
& \left.\quad+E^{-1 / 2}\left(\delta r_{a}+\frac{a}{a-1}\left\|r_{2}-r_{1}\right\|_{L^{s, s^{\prime}}\left(\mathbb{C} \backslash D_{a}\right)}\right)\right],
\end{aligned}
$$

where we used the fact that

$$
\left\|K_{j}^{ \pm}\right\|_{L^{\infty}(T)} \leq c(N, m) E^{-1 / 2} .
$$

Indeed this follows from the first equality in (4.9), equation (2.17) (derived with respect to $\partial_{z}$ and $\partial_{\bar{z}}$ ), inequality (3.14) for $\rho_{j}$, Lemma 2.1 and estimate

$$
\int_{T} \sqrt{E}\left|\lambda-\lambda^{\prime}\right|\left(1+E\left|\lambda-\lambda^{\prime}\right|^{2}\right)^{-m / 2}\left|d \lambda^{\prime}\right| \leq c E^{-1 / 2} .
$$

This inequality, as well as (3.14), also implies

$$
\sup _{\lambda \in T}\left\|\rho_{1}^{ \pm}(\lambda, \cdot)\right\|_{L^{1}(T)} \leq c(N, m) E^{-1 / 2}
$$

We also have

$$
\left\|\rho_{2}^{ \pm}-\rho_{1}^{ \pm}\right\|_{L^{2}\left(T^{2}\right)} \leq c \sqrt{E}\left\|\rho_{2}-\rho_{1}\right\|_{L^{2}\left(T^{2}\right)}
$$


which follows from the definition of $\rho\left(\lambda, \lambda^{\prime}, z\right)$ in (2.18), and

$$
\sup _{\lambda \in T}\left\|\rho_{1}(\lambda, \cdot)\right\|_{L^{1}(T)} \leq c(N, m) E^{-1 / 2} .
$$

In order to estimate the difference of $e_{j}^{ \pm}$we proceed as follows. From (4.4) we have that $e_{j}^{ \pm}$satisfies the following integral equation

$$
e_{j}^{ \pm}(\lambda)=-\frac{1}{\pi} \int_{\mathbb{C}}\left(\frac{r_{j}^{ \pm}(\zeta, z) \bar{e}_{j}(\zeta)}{\zeta-\lambda} \pm \frac{r_{j}(\zeta, z) \bar{e}_{j}^{ \pm}(\zeta)}{\zeta-\lambda}\right) d \operatorname{Re} \zeta d \operatorname{Im} \zeta
$$

which gives

$$
\begin{gathered}
\left(e_{2}^{ \pm}-e_{1}^{ \pm}\right)(\lambda)=-\frac{1}{\pi} \int_{\mathbb{C}}\left(\frac{\left(r_{2}^{ \pm}-r_{1}^{ \pm}\right)(\zeta, z) \bar{e}_{2}(\zeta)+r_{1}^{ \pm}(\zeta, z)\left(\bar{e}_{2}-\bar{e}_{1}\right)(\zeta)}{\zeta-\lambda}\right. \\
\left. \pm \frac{\left(r_{2}-r_{1}\right)(\zeta, z) \bar{e}_{2}^{ \pm}(\zeta)+r_{1}(\zeta, z)\left(\bar{e}_{2}^{ \pm}-\bar{e}_{1}^{ \pm}\right)(\zeta)}{\zeta-\lambda}\right) d \operatorname{Re} \zeta d \operatorname{Im} \zeta
\end{gathered}
$$

Using several times Hölder's inequality as well as estimates (5.5), (5.6) (see Section 5), Lemma 3.1, definition (2.15) and the $L^{\infty}$ boundedness of $e_{j}, e_{j}^{ \pm}$, we obtain

$$
\begin{aligned}
\left\|e_{2}^{ \pm}-e_{1}^{ \pm}\right\|_{L^{\infty}(T)} \leq & c(N, m)\left(\left\|r_{2}-r_{1}\right\|_{L^{s, s^{\prime}}(\mathbb{C})}\right. \\
& \left.+\sqrt{E}\left\|\left(\frac{1}{|\lambda|}+|\lambda|\right)\left|r_{2}-r_{1}\right|\right\|_{L^{s, s^{\prime}}(\mathbb{C})}\right),
\end{aligned}
$$

for $1<s<2<s^{\prime}<\infty$.

We now pass to the estimates of the $\Omega_{k}^{ \pm, j}$. Define

$$
X_{k}^{ \pm, j}=\partial_{z} X_{k}^{j} \pm \partial_{\bar{z}} X_{k}^{j}, \quad j=1,2, \quad k=1,2 .
$$

From definitions (4.7), (4.8) we have that $X_{k}^{ \pm, j}$ satisfy the following nonhomogeneous $\bar{\partial}$ equations

$$
\partial_{\bar{\lambda}} X_{k}^{ \pm, j}= \pm r_{j} \overline{X_{k}^{ \pm, j}}+r_{j}^{ \pm} \overline{X_{k}^{j}}
$$

where $X_{k}^{-, j}$ has no singularities while $X_{k}^{+, j}$ has a pole at $\lambda=\zeta$. More precisely

$$
\lim _{\lambda \rightarrow \zeta}(\lambda-\zeta) X_{1}^{+, j}(\lambda, \zeta)=1, \quad \lim _{\lambda \rightarrow \zeta}(\lambda-\zeta) X_{2}^{+, j}(\lambda, \zeta)=\frac{1}{i}
$$

We will now estimate the $X_{k}^{ \pm, j}$ using an argument of Vekua [31, Ch. III, $\S 7-8]$. Consider the following inverse of $\partial_{\bar{\lambda}}$ :

$$
\partial_{\bar{\lambda}}^{-1} f(\lambda, \zeta)=-\frac{\zeta-\lambda}{\pi} \int_{\mathbb{C}} \frac{f(\eta)}{(\eta-\lambda)(\zeta-\eta)} d \operatorname{Re} \eta d \operatorname{Im} \eta
$$


defined for $f \in L_{2}^{p}(\mathbb{C})$. It satisfies the following inequalities

$$
\begin{aligned}
\left|\partial_{\bar{\lambda}}^{-1} f(\lambda, \zeta)\right| & \leq c(p)\|f\|_{L_{2}^{p}(\mathbb{C})}, \\
\left|\partial_{\bar{\lambda}}^{-1} f(\lambda, \zeta)\right| & \leq c(p)\|f\|_{L_{2}^{p}(\mathbb{C})}|\lambda-\zeta|^{1-2 / p},
\end{aligned}
$$

which are proved in [31, Ch. III, §4]. Let

$$
w_{k}^{ \pm, j}(\lambda, \zeta)=\partial_{\bar{\lambda}}^{-1}\left( \pm r_{j}(\cdot, z) \frac{\overline{X_{k}^{ \pm, j}}(\cdot, \zeta)}{X_{k}^{ \pm, j}(\cdot, \zeta)}\right)(\lambda, \zeta) .
$$

We first consider $X_{k}^{-, j}$. Since it has no singularity, we can argue as in the proof of Proposition 4.1, and find the representation formula

$$
X_{k}^{-, j}(\lambda, \zeta)=e^{w_{k}^{-, j}(\lambda, \zeta)} \partial_{\bar{\lambda}}^{-1}\left(e^{-w_{k}^{-, j}(\cdot, \zeta)}\left(r_{j}^{-}(\cdot, z) \overline{X_{k}^{j}}(\cdot, \zeta)\right)\right)(\lambda, \zeta),
$$

which yields

$$
\left|X_{k}^{-, j}(\lambda, \zeta)\right| \leq c(N, m) E^{-(m-1) / 2},
$$

for $\lambda \in \mathbb{C}, \zeta \in T$. Indeed this follows from (4.61), the boundedness of $\left\|r_{j}\right\|_{L_{2}^{p}(\mathbb{C})}$ and the fact that

$$
\begin{aligned}
\left\|r^{ \pm}(\cdot, z)\right\|_{L_{2}^{p}(\mathbb{C})} & \leq c \sqrt{E}\left\|\left(1+|\lambda|^{2-2 / p}\right) r(\cdot, z)\right\|_{L^{p}(\mathbb{C})} \\
& \leq c(N, m) E^{-(m-1) / 2}
\end{aligned}
$$

for $p \geq 1$. We also used Hölder inequality and the estimates

$$
\begin{aligned}
\left|X_{1}^{j}(\lambda, \zeta)-\frac{1}{2(\zeta-\lambda)}\right| \leq c(N, p) \frac{1}{|\zeta-\lambda|^{2 / p}}, \\
\left|X_{2}^{j}(\lambda, \zeta)-\frac{1}{2 i(\zeta-\lambda)}\right| \leq c(N, p) \frac{1}{|\zeta-\lambda|^{2 / p}},
\end{aligned}
$$

which follow from (4.24) and (4.25).

For $X_{k}^{+, j}$ the two representation formulas hold:

$$
X_{1}^{+, j}(\lambda, \zeta)=\frac{e^{w_{1}^{+, j}(\lambda, \zeta)} \partial_{\bar{\lambda}}^{-1}\left(e^{-w_{1}^{+, j}(\cdot, \zeta)}\left(r_{j}^{+}(\cdot, z)(\zeta-\cdot) \overline{X_{1}^{j}}(\cdot, \zeta)\right)\right)(\lambda, \zeta)}{\zeta-\lambda},
$$

$$
X_{2}^{+, j}(\lambda, \zeta)=\frac{e^{w_{2}^{+, j}(\lambda, \zeta)} \partial_{\bar{\lambda}}^{-1}\left(e^{-w_{2}^{+, j}(\cdot, \zeta)}\left(r_{j}^{+}(\cdot, z) i(\zeta-\cdot) \overline{X_{2}^{j}}(\cdot, \zeta)\right)\right)(\lambda, \zeta)}{i(\zeta-\lambda)}
$$

These formulas (non-linear integral equations) are some sort of generalisations of the non-linear integral equation (7.3) in [31, Ch. III] and may be generalised to solutions of non-homogeneous $\bar{\partial}$ equations with arbitrary prescribed (analytic) singularities. 
To prove (4.69) we proceed as follows. We define $X^{\prime}(\lambda, \zeta)=(\zeta-\lambda) X_{1}^{+, j}(\lambda, \zeta)$, which is continuous and satisfies

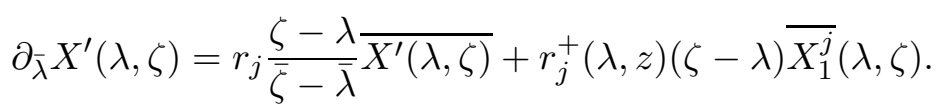

Then we have

$$
\partial_{\bar{\lambda}}\left(e^{-w_{1}^{+, j}(\lambda, \zeta)} X^{\prime}(\lambda, \zeta)\right)=e^{-w_{1}^{+, j}(\lambda, \zeta)} r_{j}^{+}(\lambda, z)(\zeta-\lambda) \overline{X_{1}^{j}}(\lambda, \zeta)
$$

It is then possible to apply $\partial_{\bar{\lambda}}$ since we have estimates (4.66)-(4.68), which guarantees that the right hand side is in $L_{2}^{p}(\mathbb{C})$, for $\zeta \in T$. The proof of (4.70) is completely analogous.

From (4.69), (4.70), as well as (4.66)-(4.68) and (4.62) we find

$$
\left|X_{k}^{+, j}(\lambda, \zeta)\right| \leq c(N, m) E^{-(m-1) / 2}|\zeta-\lambda|^{-2 / p},
$$

for $\lambda \in \mathbb{C}, \zeta \in T, j=1,2, k=1,2, p>2$. To summarize, we have obtained:

$$
\begin{aligned}
& \left|\Omega_{k}^{-, j}(\lambda, \zeta)\right| \leq c(N, m) E^{-(m-1) / 2} \\
& \left|\Omega_{k}^{+, j}(\lambda, \zeta)\right| \leq c(N, m) E^{-(m-1) / 2}|\zeta-\lambda|^{-2 / p},
\end{aligned}
$$

for $\lambda \in \mathbb{C}, \zeta \in T, j=1,2, k=1,2, p>2$.

We can now estimate the difference $\Omega_{k}^{ \pm, 2}-\Omega_{k}^{ \pm, 1}$ using similar arguments. The functions $X_{k}^{ \pm, 2}-X_{k}^{ \pm, 1}$ are continuous and satisfy

$$
\begin{aligned}
& \partial_{\bar{\lambda}}\left(X_{k}^{ \pm, 2}-X_{k}^{ \pm, 1}\right)= \pm r_{1}\left(\bar{X}_{k}^{ \pm, 2}-\bar{X}_{k}^{ \pm, 1}\right) \\
& \quad+\left(r_{2}^{ \pm}-r_{1}^{ \pm}\right) \bar{X}_{k}^{2}+r_{1}^{ \pm}\left(\bar{X}_{k}^{2}-\bar{X}_{k}^{1}\right) \pm\left(r_{2}-r_{1}\right) \bar{X}_{k}^{ \pm, 2}
\end{aligned}
$$

for $\lambda, \zeta \in \mathbb{C}, j=1,2, k=1,2$. Using the $\partial_{\bar{\lambda}}^{-1}$ defined in (4.35) and arguing as above we find

$$
\left|\left(X_{k}^{ \pm, 2}-X_{k}^{ \pm, 1}\right)(\lambda, \zeta)\right| \leq c(N)\left(J_{1}(\lambda, \zeta)+J_{2}(\lambda, \zeta)+J_{3}(\lambda, \zeta)\right)
$$

where

$$
\begin{aligned}
& J_{1}(\lambda, \zeta)=\int_{\mathbb{C}} \frac{\left|\left(r_{2}^{ \pm}-r_{1}^{ \pm}\right)(\eta, z)\right|\left|X_{k}^{2}(\eta, \zeta)\right|}{|\eta-\lambda|} d \operatorname{Re} \eta d \operatorname{Im} \eta, \\
& J_{2}(\lambda, \zeta)=\int_{\mathbb{C}} \frac{\left|r_{1}^{ \pm}(\eta, z)\right|\left|\left(X_{k}^{2}-X_{k}^{1}\right)(\eta, \zeta)\right|}{|\eta-\lambda|} d \operatorname{Re} \eta d \operatorname{Im} \eta, \\
& J_{3}(\lambda, \zeta)=\int_{\mathbb{C}} \frac{\left|\left(r_{2}-r_{1}\right)(\eta, z)\right|\left|X_{k}^{ \pm, 2}(\eta, \zeta)\right|}{|\eta-\lambda|} d \operatorname{Re} \eta d \operatorname{Im} \eta
\end{aligned}
$$


For $J_{1}$ and $J_{3}$ we argue as in the proof of Proposition 4.1. We find, for $a>1$, $p>2$,

$$
\begin{aligned}
J_{1}(\lambda, \zeta) \leq & c(N, m)\left(\delta r_{a}^{\prime}|\log | \zeta-\lambda \|\right. \\
& \left.+\frac{a}{a-1}\left\|\left(\frac{1}{|\lambda|}+|\lambda|\right)\left(r_{2}-r_{1}\right)\right\|_{L^{s, s^{\prime}}\left(\mathbb{C} \backslash D_{a}\right)}\right)
\end{aligned}
$$

for $\lambda, \zeta \in T$, where $\delta r_{a}^{\prime}=\sup _{D_{a}}\left(\frac{1}{|\lambda|}+|\lambda|\right)\left|\left(r_{2}-r_{1}\right)(\lambda)\right|, 1<s<2<s^{\prime}<$ $+\infty$. Using (4.73) for $p>4$ and (4.65), we obtain for both " \pm " cases

$$
J_{3}(\lambda, \zeta) \leq c(N, m) E^{-(m-1) / 2}\left(\delta r_{a}+\frac{a}{a-1}\left\|r_{2}-r_{1}\right\|_{L^{s, s^{\prime}}\left(\mathbb{C} \backslash D_{a}\right)}\right),
$$

for $\lambda, \zeta \in T$.

In order to estimate $J_{2}$ we start with Hölder's inequality for $q>2,1 / p+$ $1 / q=1$ :

$$
\begin{aligned}
J_{2}(\lambda, \zeta) & \leq\left\|\frac{r_{1}^{ \pm}(\cdot, z)}{|\cdot-\lambda|}\right\|_{L^{p}(\mathbb{C})}\left\|\left(X_{k}^{2}-X_{k}^{1}\right)(\cdot, \zeta)\right\|_{L^{q}(\mathbb{C})} \\
& \leq c(N, m, q) E^{-(m-1) / 2}\left\|\left(X_{k}^{2}-X_{k}^{1}\right)(\cdot, \zeta)\right\|_{L^{q}(\mathbb{C})},
\end{aligned}
$$

since we can find $r, r^{\prime}$ with $1<r^{\prime}<2<r<+\infty$ such that $1 / r+1 / r^{\prime}=1 / p$ (note that $p<2$ ) and thus

$$
\begin{aligned}
\left\|\frac{r_{1}^{ \pm}(\cdot, z)}{|\cdot-\lambda|}\right\|_{L^{q}(\mathbb{C})} \leq & \left\|r_{1}^{ \pm}(\cdot, z)\right\|_{L^{r}(|\eta|<R)}\|1 /|\cdot-\lambda|\|_{L^{r^{\prime}}(|\eta|<R)} \\
& +\left\|r_{1}^{ \pm}(\cdot, z)\right\|_{L^{r^{\prime}}(|\eta|>R)}\|1 /|\cdot-\lambda|\|_{L^{r}(|\eta|>R)} \\
\leq & c(N, m) E^{-(m-1) / 2},
\end{aligned}
$$

for $\lambda \in T$ and some fixed $R>1$. Now, since $\left(X_{k}^{2}-X_{k}^{1}\right)(\cdot, \zeta)$ is a continuous $L^{q}$ solution, $q>2$, of the non-homogeneous $\bar{\partial}$-equation (4.33) we have, from Lemma 2.3

$$
\left\|\left(X_{k}^{2}-X_{k}^{1}\right)(\cdot, \zeta)\right\|_{L^{q}(\mathbb{C})} \leq c(N)\left\|\left(r_{2}-r_{1}\right)(\cdot, z) X_{k}^{2}(\cdot, \zeta)\right\|_{L^{q^{\prime}}(\mathbb{C})},
$$

where $1 / q^{\prime}=1 / q+1 / 2$. From the fact that $q^{\prime}<2$ and $X_{k}^{2}$ satisfies (4.67), (4.68), using Hölder's inequality as above we obtain

$$
\left\|\left(X_{k}^{2}-X_{k}^{1}\right)(\cdot, \zeta)\right\|_{L^{q}(\mathbb{C})} \leq c(N)\left\|r_{2}-r_{1}\right\|_{L^{s, s^{\prime}}(\mathbb{C})},
$$

for some $1<s<2<s^{\prime}<+\infty$, therefore

$$
J_{2}(\lambda, \zeta) \leq c(N, m) E^{-(m-1) / 2}\left\|r_{2}-r_{1}\right\|_{L^{s, s^{\prime}}(\mathbb{C})},
$$


HÖLDER-LOGARITHMIC STABILITY ESTIMATE IN 2D AT POSITIVE ENERGY 27 for $\lambda, \zeta \in T$. Putting together (4.81), (4.85) and (4.82) we find

$$
\begin{aligned}
& \left\|\left(\Omega_{k}^{ \pm, 2}-\Omega_{k}^{ \pm, 1}\right)(\lambda, \cdot)\right\|_{L^{2}(T)} \\
& \leq c(N, m)\left(\delta r_{a}^{\prime}+\frac{a}{a-1}\left\|\left(\frac{1}{|\lambda|}+|\lambda|\right)\left(r_{2}-r_{1}\right)\right\|_{L^{s, s^{\prime}}\left(\mathbb{C} \backslash D_{a}\right)}\right. \\
& \left.\quad+E^{-(m-1) / 2}\left\|r_{2}-r_{1}\right\|_{L^{s, s^{\prime}}(\mathbb{C})}\right),
\end{aligned}
$$

for $\lambda \in T$, since $r_{2}-r_{1} \leq\left(\frac{1}{|\lambda|}+|\lambda|\right)\left(r_{2}-r_{1}\right)$.

We can finally put everything together and find

$$
\begin{aligned}
& \left\|K_{2}^{ \pm}-K_{1}^{ \pm}\right\|_{L^{2}(T)} \leq c(N, m)\left[E^{1 / 2}\left\|\rho_{2}-\rho_{1}\right\|_{L^{2}\left(T^{2}\right)}\right. \\
& +\left\|\left(\frac{1}{|\lambda|}+|\lambda|\right)\left(r_{2}-r_{1}\right)\right\|_{L^{s, s^{\prime}}(\mathbb{C})} \\
& \left.\quad+E^{-1}\left(\delta r_{a}^{\prime}+\frac{a}{a-1}\left\|\left(\frac{1}{|\lambda|}+|\lambda|\right)\left(r_{2}-r_{1}\right)\right\|_{L^{s, s^{\prime}}\left(\mathbb{C} \backslash D_{a}\right)}\right)\right],
\end{aligned}
$$

which finishes the proof of Proposition 4.2 .

\section{Proof of Theorem 1.1}

We start from formula (4.15) and estimate the differences $A_{2}-A_{1}, B_{2}-B_{1}$ and $C_{2}-C_{1}$ separately. We have

$$
\begin{aligned}
A_{2}-A_{1}= & \frac{1}{\pi} \int_{\mathbb{C}}\left[-\frac{i \sqrt{E}}{2}\left(\frac{1}{\zeta}+\bar{\zeta}\right)\left(\left(r_{2}-r_{1}\right) \overline{e_{2}}+r_{1}\left(\overline{e_{2}}-\overline{e_{1}}\right)\right)\right. \\
& \left.+\left(r_{2}-r_{1}\right) \overline{\partial_{\bar{z}} e_{2}}+r_{1} \overline{\left(\partial_{\bar{z}} e_{2}-\partial_{\bar{z}} e_{1}\right)}\right] d \operatorname{Re} \zeta d \operatorname{Im} \zeta
\end{aligned}
$$

Using several times Hölder's inequality (2.30), we find

$$
\begin{aligned}
& \left|A_{2}-A_{1}\right| \leq \frac{1}{\pi}\left[\frac { \sqrt { E } } { 2 } \left(\left\|\left(\frac{1}{\zeta}+\bar{\zeta}\right)\left(r_{2}-r_{1}\right)\right\|_{L^{1}(\mathbb{C})}\left\|e_{2}(z, \cdot)\right\|_{L^{\infty}(\mathbb{C})}\right.\right. \\
& \left.\quad+\left\|\left(\frac{1}{\zeta}+\bar{\zeta}\right) r_{1}\right\|_{L^{\tilde{p}^{\prime}}(\mathbb{C})}\left\|e_{2}(z, \cdot)-e_{2}(z, \cdot)\right\|_{L^{\tilde{p}}(\mathbb{C})}\right) \\
& \quad+\left\|r_{2}-r_{1}\right\|_{L^{p}(\mathbb{C})}\left\|\partial_{\bar{z}} e_{2}(z, \cdot)\right\|_{L^{p^{\prime}}(\mathbb{C})} \\
& \left.\quad+\left\|r_{1}\right\|_{L^{\tilde{p}^{\prime}}(\mathbb{C})}\left\|\partial_{\bar{z}} e_{2}(z, \cdot)-\partial_{\bar{z}} e_{1}(z, \cdot)\right\|_{L^{\tilde{p}}(\mathbb{C})}\right],
\end{aligned}
$$

for $1<p<2, \tilde{p}$ such that $1 / \tilde{p}=1 / p-1 / 2$ and $1 / p+1 / p^{\prime}=1 / \tilde{p}+1 / \tilde{p}^{\prime}=1$. 
In order to estimate $\left\|e_{2}(z, \cdot)-e_{2}(z, \cdot)\right\|_{L^{\tilde{p}}}$ and $\left\|\partial_{\bar{z}} e_{2}(z, \cdot)-\partial_{\bar{z}} e_{1}(z, \cdot)\right\|_{L^{\tilde{p}}}$ we just remark that from the definition (4.4) $e_{j}(z, \lambda)$ satisfies

$$
\frac{\partial}{\partial \bar{\lambda}} e_{j}(z, \lambda)=r_{j}(z, \lambda) \overline{e_{j}(z, \lambda)}, \quad j=1,2,
$$

for all $\lambda \in \mathbb{C}$, with $\lim _{\lambda \rightarrow \infty} e_{j}(z, \lambda)=1$. The operator $\partial_{\bar{\lambda}}^{-1}$ defined in (4.35), which intervene in the integral equation defining $e_{j}(\lambda)$, satisfies the estimate

$$
\left|\partial_{\bar{\lambda}}^{-1} f(\lambda)\right| \leq c(p)\|f\|_{L_{2}^{p}(\mathbb{C})}|\lambda|^{2 / p-1}, \quad \text { for }|\lambda|>1, \quad p>2,
$$

which is proved in [31, Ch. III, (4.16)] (see (4.10) for the definition of $L_{2}^{p}(\mathbb{C})$ ). As already stated at the beginning of Section 4 since $r_{j}(\lambda) \in L_{2}^{p}(\mathbb{C})$, equation (4.4) is uniquely solved in $L_{0}^{q}(\mathbb{C})(p /(p-1) \leq q<2)$ and in addition $e(\lambda)$ is continuous (see [31]). Then $e(\lambda)$ is $L^{\infty}(\mathbb{C})$ and since $r_{j}(\lambda)$ is bounded in $L_{2}^{p}(\mathbb{C})$ for every $p \geq 1$, we obtain

$$
|e(\lambda)-1| \leq c(N, p)|\lambda|^{2 / p-1}, \quad|\lambda|>1,
$$

for every $p>2$, which yields $\|e(\cdot)-1\|_{L^{q}(\mathbb{C})} \leq c(N, q)$, for every $q>2$ (since $e$ is continuous on $\mathbb{C}$ ). The same kind of argument yields

$$
\left\|\partial_{\bar{z}} e(\cdot)\right\|_{L^{q}(\mathbb{C})} \leq c(N, q), \quad\left\|\partial_{z} e(\cdot)\right\|_{L^{q}(\mathbb{C})} \leq c(N, q), \quad \text { for any } q>2 .
$$

Thus it is possible to use the same ideas as in [30, Lemma 4.1] to estimate $e_{j}$ as follows:

$$
\begin{gathered}
\sup _{z \in \mathbb{C}}\left\|e_{2}(z, \cdot)-e_{1}(z, \cdot)\right\|_{L^{\tilde{p}}(\mathbb{C})} \leq c(D, N, p, m)\left\|r_{2}-r_{1}\right\|_{L^{p}(\mathbb{C})}, \\
\sup _{z \in \mathbb{C}}\left\|\nabla e_{2}(z, \cdot)-\nabla e_{1}(z, \cdot)\right\|_{L^{\tilde{p}}(\mathbb{C})} \leq c(D, N, p, m)\left[\left\|r_{2}-r_{1}\right\|_{L^{p}(\mathbb{C})}\right. \\
\left.\quad+\sqrt{E}\left(\left\|\left(|\lambda|+\frac{1}{|\lambda|}\right)\left|r_{2}-r_{1}\right|\right\|_{L^{p}(\mathbb{C})}+\left\|r_{2}-r_{1}\right\|_{L^{p}(\mathbb{C})}\right)\right],
\end{gathered}
$$

with $p$ and $\tilde{p}$ defined above and $\nabla$ is taken with respect to $z$. The proof of (5.5) is exactly the same as that of the first estimate of [30, Lemma 4.1] while for (5.6) the only differences are in some signs, due to (2.15), and do not affect the result.

From Lemma 2.2 we find

$$
\begin{aligned}
\left\|\left(\frac{1}{\zeta}+\bar{\zeta}\right) r_{1}\right\|_{L^{\tilde{p}^{\prime}}(\mathbb{C})} & \leq c\left(N, m, p^{\prime}\right) E^{-m / 2} \\
\left\|r_{1}\right\|_{L^{\tilde{p}^{\prime}}(\mathbb{C})} & \leq c\left(N, m, p^{\prime}\right) E^{-m / 2} .
\end{aligned}
$$


HÖLDER-LOGARITHMIC STABILITY ESTIMATE IN 2D AT POSITIVE ENERGY 29

Combining estimates (5.5)-(5.8) with (5.1) we find, for a fixed $p \in] 1,2[$,

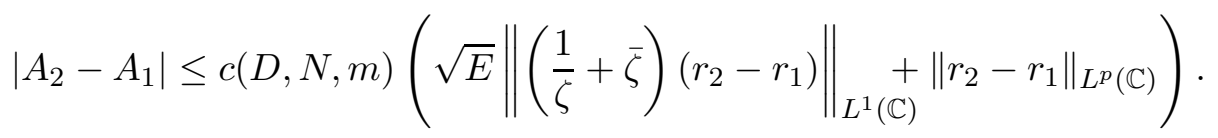

Then, using Proposition 3.5 we obtain

$$
\begin{aligned}
\left|A_{2}-A_{1}\right| \leq & c(D, N, m)\left[E^{-1 / 2}\left(E^{1 / 2}+\kappa \log \left(3+\delta^{-1}\right)\right)^{-(m-2)}\right. \\
& \left.+\delta\left(3+\delta^{-1}\right)^{2 \kappa(l+1)}\right]
\end{aligned}
$$

for $\kappa$ and $\delta$ as in the statement. We now pass to $B_{2}-B_{1}$, which is given by

$$
\begin{aligned}
B_{2}-B_{1}= & \frac{1}{2 \pi i} \int_{|\zeta|=1}\left\{\left(\partial_{z} K_{2}(\zeta)-\partial_{z} K_{1}(\zeta)\right)\right. \\
& \times\left[-1+\frac{1}{\pi} \int_{\mathbb{C}} r_{2}(\lambda, z) \overline{\Omega_{2}^{2}(\lambda, \zeta)} d \operatorname{Re} \lambda d \operatorname{Im} \lambda\right] \\
& +\partial_{z} K_{1}(\zeta)\left[\frac{1}{\pi} \int_{\mathbb{C}}\left(r_{2}-r_{1}\right)(\lambda, z) \overline{\Omega_{2}^{2}(\lambda, \zeta)} d \operatorname{Re} \lambda d \operatorname{Im} \lambda\right. \\
& \left.+\frac{1}{\pi} \int_{\mathbb{C}} r_{1}(\lambda, z)\left(\overline{\Omega_{2}^{2}(\lambda, \zeta)}-\overline{\Omega_{2}^{1}(\lambda, \zeta)}\right) d \operatorname{Re} \lambda d \operatorname{Im} \lambda\right] \\
& +\frac{\left(K_{2}-K_{1}\right)(\zeta)}{\pi} \int_{\mathbb{C}} r_{2}(\lambda, z)\left[-\frac{i \sqrt{E}}{2}\left(\frac{1}{\lambda}+\bar{\lambda}\right) \overline{\Omega_{2}^{2}(\lambda, \zeta)}\right. \\
& \left.+\overline{\partial_{\bar{z}} \Omega_{2}^{2}(\lambda, \zeta)}\right] d \operatorname{Re} \lambda d \operatorname{Im} \lambda+\frac{K_{1}(\zeta)}{2 \pi} \int_{\mathbb{C}}\left(r_{2}-r_{1}\right)(\lambda, z) \\
& \times\left[-\frac{i \sqrt{E}}{2}\left(\bar{\lambda}+\frac{1}{\lambda}\right) \overline{\Omega_{2}^{2}(\lambda, \zeta)}+\overline{\partial_{\bar{z}} \Omega_{2}^{2}(\lambda, \zeta)}\right] \\
& +r_{1}(\lambda, z)\left[-\frac{i \sqrt{E}}{2}\left(\bar{\lambda}+\frac{1}{\lambda}\right)\left(\overline{\Omega_{2}^{2}(\lambda, \zeta)}-\overline{\Omega_{2}^{1}(\lambda, \zeta)}\right)\right. \\
& \left.\left.+\overline{\partial_{\bar{z}} \Omega_{2}^{2}(\lambda, \zeta)}-\overline{\partial_{\bar{z}} \Omega_{2}^{1}(\lambda, \zeta)}\right] d \operatorname{Re} \lambda d \operatorname{Im} \lambda\right\} d \zeta .
\end{aligned}
$$

This yields

$$
\begin{aligned}
\left|B_{2}-B_{1}\right| \leq & \frac{1}{2 \pi}\left\|\partial_{z} K_{2}-\partial_{z} K_{1}\right\|_{L^{2}(T)} \\
& \times\left\|-1+\frac{1}{\pi} \int_{\mathbb{C}} r_{2}(\lambda, z) \overline{\Omega_{2}^{2}(\lambda, \cdot)} d \operatorname{Re} \lambda d \operatorname{Im} \lambda\right\|_{L^{2}(T)} \\
& +\left\|\partial_{z} K_{1}\right\|_{L^{2}(T)} \| \frac{1}{\pi} \int_{\mathbb{C}}\left(r_{2}-r_{1}\right)(\lambda, z) \overline{\Omega_{2}^{2}(\lambda, \zeta)} d \operatorname{Re} \lambda d \operatorname{Im} \lambda \\
& +\frac{1}{\pi} \int_{\mathbb{C}} r_{1}(\lambda, z)\left(\overline{\Omega_{2}^{2}(\lambda, \cdot)}-\overline{\Omega_{2}^{1}(\lambda, \zeta)}\right) d \operatorname{Re} \lambda d \operatorname{Im} \lambda \|_{L^{2}(T)} \\
& +\frac{\left\|K_{2}-K_{1}\right\|_{L^{2}(T)}}{\pi} \| \int_{\mathbb{C}} r_{2}(\lambda, z)\left[-\frac{i \sqrt{E}}{2}\left(\frac{1}{\lambda}+\bar{\lambda}\right) \overline{\Omega_{2}^{2}(\lambda, \cdot)}\right.
\end{aligned}
$$




$$
\begin{aligned}
& \left.+\overline{\partial_{\bar{z}} \Omega_{2}^{2}(\lambda, \cdot)}\right] d \operatorname{Re} \lambda d \operatorname{Im} \lambda\left\|_{L^{2}(T)}+\frac{\left\|K_{1}\right\|_{L^{2}(T)}}{2 \pi}\right\| \int_{\mathbb{C}}\left(r_{2}-r_{1}\right)(\lambda, z) \\
& \times\left[-\frac{i \sqrt{E}}{2}\left(\bar{\lambda}+\frac{1}{\lambda}\right) \overline{\Omega_{2}^{2}(\lambda, \cdot)}+\overline{\partial_{\bar{z}} \Omega_{2}^{2}(\lambda, \cdot)}\right] \\
& +r_{1}(\lambda, z)\left[-\frac{i \sqrt{E}}{2}\left(\bar{\lambda}+\frac{1}{\lambda}\right)\left(\overline{\Omega_{2}^{2}(\lambda, \cdot)}-\overline{\Omega_{2}^{1}(\lambda, \cdot)}\right)\right. \\
& \left.+\overline{\partial_{\bar{z}} \Omega_{2}^{2}(\lambda, \cdot)}-\overline{\partial_{\bar{z}} \Omega_{2}^{1}(\lambda, \cdot)}\right] d \operatorname{Re} \lambda d \operatorname{Im} \lambda \|_{L^{2}(T)} .
\end{aligned}
$$

Using Hölder's inequality, Lemma 3.1, estimates (4.25), (4.49), (4.84), (4.74), (4.75), (4.32) we find

$$
\begin{aligned}
\left|B_{2}-B_{1}\right| \leq & c(N, m)\left[\left\|\partial_{z} K_{2}-\partial_{z} K_{1}\right\|_{L^{2}(T)}+\left\|\left(\frac{1}{|\lambda|}+|\lambda|\right)\left(r_{2}-r_{1}\right)\right\|_{L^{s, s^{\prime}}(\mathbb{C})}\right. \\
& \left.+\frac{\left\|K_{2}-K_{1}\right\|_{L^{2}(T)}}{\sqrt{E}^{m-1}}+\frac{\sup _{\zeta \in T}\left\|\partial_{\bar{z}} \Omega_{2}^{2}(\cdot, \zeta)-\partial_{\bar{z}} \Omega_{2}^{1}(\cdot, \zeta)\right\|_{L^{q}(\mathbb{C})}}{\sqrt{E}^{m-1}}\right],
\end{aligned}
$$

for some $1<s<2<s^{\prime}<+\infty, q>2$. We estimate the last term using Lemma 2.3. Since $\left(X_{k}^{ \pm, 2}-X_{k}^{ \pm, 1}\right)(\cdot, \zeta)$, defined in (4.57), is a continuous $L^{q}$ solution, $q>2$, of the non-homogeneous $\bar{\partial}$-equation (4.76) we have, from Lemma 2.3 ,

$$
\begin{aligned}
\left\|\left(X_{k}^{ \pm, 2}-X_{k}^{ \pm, 1}\right)(\cdot, \zeta)\right\|_{L^{q}(\mathbb{C})} \leq & c(N)\left(\left\|\left(r_{2}^{ \pm}-r_{1}^{ \pm}\right)(\cdot, z) X_{k}^{2}(\cdot, \zeta)\right\|_{L^{q^{\prime}}(\mathbb{C})}\right. \\
& +\left\|r_{1}^{ \pm}(\cdot, z)\left(X_{k}^{2}-X_{k}^{1}\right)(\cdot, \zeta)\right\|_{L^{q^{\prime}}(\mathbb{C})} \\
& \left.+\left\|\left(r_{2}-r_{1}\right)(\cdot, z) X_{k}^{ \pm, 2}(\cdot, \zeta)\right\|_{L^{q^{\prime}}(\mathbb{C})}\right) \\
\leq & c(N, m)\left(\sqrt{E}\left\|\left(\frac{1}{|\lambda|}+|\lambda|\right)\left(r_{2}-r_{1}\right)\right\|_{L^{s, s^{\prime}}(\mathbb{C})}\right. \\
& +\sqrt{E}^{-(m-1)}\left\|\left(X_{k}^{2}-X_{k}^{1}\right)(\cdot, \zeta)\right\|_{L^{r}(\mathbb{C})} \\
& \left.+\sqrt{E}^{-(m-1)}\left\|\left(r_{2}-r_{1}\right)(\cdot, z)\right\|_{L^{s, s^{\prime}}(\mathbb{C})}\right)
\end{aligned}
$$

where $1 / q^{\prime}=1 / q+1 / 2$ and $r>2$. Here we used several times Hölder's inequality, the fact that $q^{\prime}<2$ and that $X_{k}^{j}, X_{k}^{ \pm, j}$ satisfy (4.67), (4.68) and (4.73), (4.65). From (4.84) and the fact that $r_{2}-r_{1} \leq\left(\frac{1}{|\lambda|}+|\lambda|\right)\left(r_{2}-r_{1}\right)$ we obtain

$$
\left\|\left(X_{k}^{ \pm, 2}-X_{k}^{ \pm, 1}\right)(\cdot, \zeta)\right\|_{L^{q}(\mathbb{C})} \leq c(N, m) \sqrt{E}\left\|\left(\frac{1}{|\lambda|}+|\lambda|\right)\left(r_{2}-r_{1}\right)\right\|_{L^{s, s^{\prime}}(\mathbb{C})},
$$


HÖLDER-LOGARITHMIC STABILITY ESTIMATE IN 2D AT POSITIVE ENERGY 31 which yields

$$
\left\|\left(\nabla \Omega_{k}^{2}-\nabla \Omega_{k}^{1}\right)(\cdot, \zeta)\right\|_{L^{q}(\mathbb{C})} \leq c(N, m) \sqrt{E}\left\|\left(\frac{1}{|\lambda|}+|\lambda|\right)\left(r_{2}-r_{1}\right)\right\|_{L^{s, s^{\prime}}(\mathbb{C})},
$$

for some $1<s<2<s^{\prime}<+\infty, q>2$.

Now, Propositions 4.1 and 4.2 as well as estimate (5.10) gives

$$
\begin{aligned}
\left|B_{2}-B_{1}\right| \leq & c(N, m)\left[E^{1 / 2}\left\|\rho_{2}-\rho_{1}\right\|_{L^{2}\left(T^{2}\right)}\right. \\
& +\left\|\left(\frac{1}{|\lambda|}+|\lambda|\right)\left(r_{2}-r_{1}\right)\right\|_{L^{s, s^{\prime}}(\mathbb{C})} \\
& \left.+E^{-1}\left(\delta r_{a}^{\prime}+\frac{a}{a-1}\left\|\left(\frac{1}{|\lambda|}+|\lambda|\right)\left(r_{2}-r_{1}\right)\right\|_{L^{s, s^{\prime}}\left(\mathbb{C} \backslash D_{a}\right)}\right)\right] .
\end{aligned}
$$

From Lemma 3.2 and (3.30) we find

$$
\delta r_{a}^{\prime} \leq c(D, N, m) e^{4(l+1) \sqrt{E}(a-1)} \delta .
$$

Like in the proof of Proposition 3.5 we define

$$
a=1+\frac{\kappa \log \left(3+\delta^{-1}\right)}{\sqrt{E}},
$$

for $\kappa<1 / 4(l+1)$. Note that

$$
\frac{a}{a-1}=1+\frac{\sqrt{E}}{\kappa \log \left(3+\delta^{-1}\right)} \leq 1+\sqrt{E},
$$

for $\delta<\frac{1}{e^{1 / \kappa}-3}$. Repeating the proof of Proposition 3.5 we obtain

$$
\begin{aligned}
& \delta r_{a}^{\prime}+\frac{a}{a-1}\left\|\left(\frac{1}{|\lambda|}+|\lambda|\right)\left(r_{2}-r_{1}\right)\right\|_{L^{s, s^{\prime}}\left(\mathbb{C} \backslash D_{a}\right)} \\
& \quad \leq c(D, N, m)\left(\delta\left(3+\delta^{-1}\right)^{4 \kappa(l+1)}+E^{-\frac{1}{2}}\left(E^{\frac{1}{2}}+\kappa \log \left(3+\delta^{-1}\right)\right)^{-(m-2)}\right),
\end{aligned}
$$

for $\delta<\frac{1}{e^{1 / \kappa}-3}, \kappa<1 / 4(l+1)$. Then, using Propositions 3.3 and 3.3 we get

$$
\begin{aligned}
\left|B_{2}-B_{1}\right| \leq & c(D, N, m)\left(\sqrt{E} \delta\left(3+\delta^{-1}\right)^{4 \kappa(l+1)}\right. \\
& \left.+E^{-1}\left(E^{\frac{1}{2}}+\kappa \log \left(3+\delta^{-1}\right)\right)^{-(m-2)}\right),
\end{aligned}
$$

for $\delta<\frac{1}{e^{1 / \kappa}-3}, \kappa<1 / 4(l+1), E>E_{4}$. 
We need now to estimate $C_{2}-C_{1}$, which can be written as follows:

$$
\begin{aligned}
C_{2}-C_{1}= & -\frac{1}{2 \pi i} \int_{|\zeta|=1}\left\{\left(\overline{\partial_{\bar{z}} K_{2}-\partial_{\bar{z}} K_{1}}\right)(\zeta)\right. \\
& \times\left[\frac{1}{\pi} \int_{\mathbb{C}} r_{2}(\lambda, z) \overline{\Omega_{1}^{2}(\lambda, \zeta)} d \operatorname{Re} \lambda d \operatorname{Im} \lambda\right] \\
& +\overline{\partial_{\bar{z}} K_{1}}(\zeta)\left[\frac{1}{\pi} \int_{\mathbb{C}}\left(r_{2}-r_{1}\right)(\lambda, z) \overline{\Omega_{1}^{2}(\lambda, \zeta)} d \operatorname{Re} \lambda d \operatorname{Im} \lambda\right. \\
& \left.+\frac{1}{\pi} \int_{\mathbb{C}} r_{1}(\lambda, z)\left(\overline{\Omega_{1}^{2}(\lambda, \zeta)}-\overline{\Omega_{1}^{1}(\lambda, \zeta)}\right) d \operatorname{Re} \lambda d \operatorname{Im} \lambda\right] \\
& +\frac{\left(\overline{K_{2}-K_{1}}\right)(\zeta)}{\pi} \int_{\mathbb{C}} r_{2}(\lambda, z)\left[-\frac{i \sqrt{E}}{2}\left(\frac{1}{\lambda}+\bar{\lambda}\right) \overline{\Omega_{1}^{2}(\lambda, \zeta)}\right. \\
& \left.+\overline{\partial_{\bar{z}} \Omega_{1}^{2}(\lambda, \zeta)}\right] d \operatorname{Re} \lambda d \operatorname{Im} \lambda+\frac{\overline{K_{1}}(\zeta)}{2 \pi} \int_{\mathbb{C}}\left(r_{2}-r_{1}\right)(\lambda, z) \\
& \times\left[-\frac{i \sqrt{E}}{2}\left(\bar{\lambda}+\frac{1}{\lambda}\right) \overline{\Omega_{1}^{2}(\lambda, \zeta)}+\overline{\partial_{\bar{z}} \Omega_{1}^{2}(\lambda, \zeta)}\right] \\
& +r_{1}(\lambda, z)\left[-\frac{i \sqrt{E}}{2}\left(\bar{\lambda}+\frac{1}{\lambda}\right)\left(\overline{\Omega_{1}^{2}(\lambda, \zeta)}-\overline{\Omega_{1}^{1}(\lambda, \zeta)}\right)\right. \\
& \left.\left.+\overline{\partial_{\bar{z}} \Omega_{1}^{2}(\lambda, \zeta)}-\overline{\partial_{\bar{z}} \Omega_{1}^{1}(\lambda, \zeta)}\right] d \operatorname{Re} \lambda d \operatorname{Im} \lambda\right\} d \zeta .
\end{aligned}
$$

We proceed exactly as for $B_{2}-B_{1}$ and we find

$$
\begin{aligned}
\left|C_{2}-C_{1}\right| \leq & c(N, m)\left[\left\|\left(\frac{1}{|\lambda|}+|\lambda|\right)\left(r_{2}-r_{1}\right)\right\|_{L^{s, s^{\prime}}(\mathbb{C})}\right. \\
& \left.+\frac{\left\|K_{2}-K_{1}\right\|_{L^{2}(T)}}{\sqrt{E}^{m-1}}+\frac{\left\|\partial_{\bar{z}} K_{2}-\partial_{\bar{z}} K_{1}\right\|_{L^{2}(T)}}{\sqrt{E}^{m}}\right],
\end{aligned}
$$

for some $1<s<2<s^{\prime}<+\infty$. Here we used again Hölder's inequality as well as Lemma 3.1, estimates (4.24), (4.49), (4.84), (4.74), (4.75) and (5.10). Using Propositions 4.1 and 4.2 with $a$ defined in (5.12) and arguing as for $B_{2}-B_{1}$ we obtain, with Propositions 3.3 and 3.5 .

$$
\begin{aligned}
\left|C_{2}-C_{1}\right| \leq & c(D, N, m)\left(\delta\left(3+\delta^{-1}\right)^{4 \kappa(l+1)}\right. \\
& \left.+E^{-1}\left(E^{\frac{1}{2}}+\kappa \log \left(3+\delta^{-1}\right)\right)^{-(m-2)}\right)
\end{aligned}
$$

for $\delta<\frac{1}{e^{1 / \kappa}-3}, \kappa<1 / 4(l+1), E>E_{4}$. 
We can now put estimates (5.9), (5.15) and (5.16) together and from (4.15) find

$$
\begin{aligned}
\left\|v_{2}-v_{1}\right\|_{L^{\infty}(D)} \leq & c(D, N, m)\left(E \delta\left(3+\delta^{-1}\right)^{4 \kappa(l+1)}\right. \\
& \left.+\left(E^{\frac{1}{2}}+\kappa \log \left(3+\delta^{-1}\right)\right)^{-(m-2)}\right),
\end{aligned}
$$

for $\delta<\frac{1}{e^{1 / \kappa}-3}, \kappa<1 / 4(l+1), E>E_{1}=\max \left(E_{0}, E_{2}, E_{3}, E_{4}\right)$. Now, for every $0<\tau<1$ there is a $0<\kappa<1 / 4(l+1)$ such that $\tau=1-4 \kappa(l+1)$. Then we have

$\left\|v_{2}-v_{1}\right\|_{L^{\infty}(D)} \leq c(D, N, m)\left(E \delta^{\tau}+\left(E^{\frac{1}{2}}+(1-\tau) \log \left(3+\delta^{-1}\right)\right)^{-(m-2)}\right)$,

for $\delta<\delta_{\tau}$ and $E>E_{1}$. This finishes the proof of Theorem 1.1.

\section{REFERENCES}

[1] Alessandrini, G., Stable determination of conductivity by boundary measurements, Appl. Anal. 27, 1988, no. 1, 153-172.

[2] Alessandrini, G., Vessella, S., Lipschitz stability for the inverse conductivity problem, Adv. in Appl. Math. 35, 2005, no. 2, 207-241.

[3] Bacchelli, V., Vessella, S., Lipschitz stability for a stationary 2D inverse problem with unknown polygonal boundary, Inverse Problems 22, no. 5, 1627.

[4] Barceló, J. A., Barceló, T., Ruiz, A., Stability of the inverse conductivity problem in the plane for less regular conductivities, J. Diff. Equations 173, 2001, 231-270.

[5] Beretta, E., de Hoop, M. V., Qiu, L., Lipschitz Stability of an Inverse Boundary Value Problem for a Schrödinger-Type Equation, SIAM J. Math. Anal. 45, 2013, no. 2, 679-699.

[6] Bourgeois, L., A remark on Lipschitz stability for inverse problems, C. R. Acad. Sci. Paris, Ser. I, to appear.

[7] Bukhgeim, A. L., Recovering a potential from Cauchy data in the two-dimensional case, J. Inverse Ill-Posed Probl. 16, 2008, no. 1, 19-33.

[8] Calderón, A. P., On an inverse boundary problem, Seminar on Numerical Analysis and its Applications to Continuum Physics, Soc. Brasiliera de Matematica, Rio de Janeiro, 1980, 61-73.

[9] Faddeev, L. D., Growing solutions of the Schrödinger equation, Dokl. Akad. Nauk SSSR 165, 1965, no. 3, 514-517.

[10] Faddeev, L. D., The inverse problem in the quantum theory of scattering. II, Current Problems in Mathematics [in Russian], Vol. 3, Akad. Nauk SSSR, Vsesoyuznyi Inst. Nauchnoi i Tekhnicheskoi Informatsii, Moscow, 1974, 93-180.

[11] Gel'fand, I. M., Some aspects of functional analysis and algebra, Proceedings of the International Congress of Mathematicians, Amsterdam, 1954, 1, 253-276. Erven P. Noordhoff N.V., Groningen; North-Holland Publishing Co., Amsterdam.

[12] Grinevich, P. G., Manakov, S. V., Inverse problem of scattering theory for the twodimensional Schrödinger operator, the $\bar{\partial}$-method and nonlinear equations, (Russian) Funktsional. Anal. i Prilozhen. 20, 1986, no. 2, 14-24, 96. 
[13] Isaev, M. I., Exponential instability in the Gel'fand inverse problem on the energy intervals, J. Inverse Ill-Posed Probl. 19, 2011, no. 3, 453-472.

[14] Isaev, M. I., Instability in the Gel'fand inverse problem at high energies, Applicable Analysis, 2012, doi:10.1080/00036811.2012.731501.

[15] Isaev, M. I., Energy and regularity dependent stability estimates for near-field inverse scattering in multidimensions, Journal of Mathematics 2013, 2013, Article ID 318154, 10 pages.

[16] Isaev, M. I., Novikov, R. G., Stability estimates for determination of potential from the impedance boundary map, Algebra and Analysis 25, 2013, no. 1, 37-63.

[17] Isaev, M. I., Novikov, R. G., Energy and regularity dependent stability estimates for the Gel'fand inverse problem in multidimensions, J. of Inverse and Ill-posed Probl. 20, 2012, no. 3, 313-325.

[18] Isakov, V., Increasing stability for the Schrödinger potential from the Dirichlet-toNeumann map, Discrete Contin. Dyn. Syst. Ser. S 4, 2011, no. 3, 631-640.

[19] Mandache, N., Exponential instability in an inverse problem of the Schrödinger equation, Inverse Problems 17, 2001, no. 5, 1435-1444.

[20] Novikov, R. G., Multidimensional inverse spectral problem for the equation $-\Delta \psi+$ $(v(x)-E u(x)) \psi=0$, Funkt. Anal. i Pril. 22, 1988, no. 4, 11-22 (in Russian); English Transl.: Funct. Anal. and Appl. 22, 1988, no. 4, 263-272.

[21] Novikov, R. G., The inverse scattering problem on a fixed energy level for the twodimensional Schrödinger operator, J. Funct. Anal. 103, 1992, no. 2, 409-463.

[22] Novikov, R. G., Approximate solution of the inverse problem of quantum scattering theory with fixed energy in dimension 2, (Russian) Tr. Mat. Inst. Steklova 225, 1999, Solitony Geom. Topol. na Perekrest., 301-318; translation in Proc. Steklov Inst. Math. 225, 1999, no. 2, 285-302.

[23] Novikov, R. G., Formulae and equations for finding scattering data from the Dirichletto-Neumann map with nonzero background potential, Inv. Problems 21, 2005, no. 1, $257-270$

[24] Novikov, R. G., The $\bar{\partial}$-approach to monochromatic inverse scattering in three dimensions, J. Geom. Anal. 18, 2008, no. 2, 612-631.

[25] Novikov, R. G., New global stability estimates for the Gel'fand-Calderon inverse problem, Inv. Problems 27, 2011, no. 1, 015001.

[26] Novikov, R. G., Novikova, N. N., On stable determination of potential by boundary measurements, ESAIM: Proc. 26, 2009, 94-99.

[27] Novikov, R. G., Santacesaria, M., A global stability estimate for the Gel'fand-Calderón inverse problem in two dimensions, J. Inverse Ill-Posed Probl. 18, 2010, no. 7, 765785 .

[28] Novikov, R. G., Santacesaria, M., Monochromatic reconstruction algorithms for twodimensional multi-channel inverse problems, Int. Math. Res. Notices 2013, 2013, no. 6, 1205-1229.

[29] Santacesaria, M., New global stability estimates for the Calderón problem in two dimensions, J. Inst. Math. Jussieu 12, 2013, no. 03, 553-569.

[30] Santacesaria, M., Stability estimates for an inverse problem for the Schrödinger equation at negative energy in two dimensions, Applicable Analysis, 2012, doi:10.1080/00036811.2012.698006.

[31] Vekua, I. N., Generalized Analytic Functions, Pergamon Press Ltd. 1962. 
HÖLDER-LOGARITHMIC STABILITY ESTIMATE IN 2D AT POSITIVE ENERGY 35 (M. Santacesaria) Centre de Mathématiques Appliquées - UMr 7641, École Polytechnique, 91128, Palaiseau, France

E-mail address: santacesaria@cmap.polytechnique.fr 